\title{
MOMENTS OF THE DOUBLY TRUNCATED SELECTION ELLIPTICAL DISTRIBUTIONS WITH EMPHASIS ON THE UNIFIED MULTIVARIATE SKEW- $t$ DISTRIBUTION
}

\author{
Christian E. Galarza \\ Departamento de Estadística \\ Escuela Superior Politecnica del Litoral \\ Guayaquil, Ecuador \\ chedgala@espol.edu.ec
}

\author{
Larissa A. Matos \\ Departamento de Estatística \\ Universidade Estadual de Campinas \\ Campinas, Brazil \\ larissam@unicamp.br
}

\author{
Victor H. Lachos \\ Department of Statistics \\ University of Connecticut \\ Storrs CT 06269, U.S.A. \\ hlachos@uconn.edu
}

July 30,2020

\begin{abstract}
In this paper, we compute doubly truncated moments for the selection elliptical (SE) class of distributions, which includes some multivariate asymmetric versions of well-known elliptical distributions, such as, the normal, Student's $t$, slash, among others. We address the moments for doubly truncated members of this family, establishing neat formulation for high order moments as well as for its first two moments. We establish sufficient and necessary conditions for the existence of these truncated moments. Further, we propose optimized methods able to deal with extreme setting of the parameters, partitions with almost zero volume or no truncation which are validated with a brief numerical study. Finally, we present some results useful in interval censoring models. All results has been particularized to the unified skew- $t$ (SUT) distribution, a complex multivariate asymmetric heavy-tailed distribution which includes the extended skew- $t$ (EST), extended skew-normal (ESN), skew- $t$ (ST) and skew-normal (SN) distributions as particular and limiting cases.
\end{abstract}

Keywords Censored regression models · Elliptical distributions · Selection distributions · Truncated distributions . Truncated moments

\section{Introduction}

Truncated moments have been a topic of high interest in the statistical literature, whose possible applications are wide, from simple to complex statistical models as survival analysis, censored data models, and in the most varied areas of applications such as agronomy, insurance, finance, biology, among others. These areas have data whose inherent characteristics lead to the use of methods that involve these truncated moments, such as restricted responses to a certain interval, partial information such as censoring (which may be left, right or interval), missing, among others. The need to have more flexible models that incorporate features such as asymmetry and robustness, has led to the exploration of this area in last years. From the first two one-sided truncated moments for the normal distribution, useful in Tobin's model ([1]), its evolution led to its extension to the multivariate case $([2])$, double truncation $([\overline{3}])$, heavy tails when considering the Student's $t$ bivariate case in [4], and finally the first two moments for the multivariate Student's $t$ case in [5]. Besides the interval-type truncation in cases before, [6] considers an interesting non-centered ellipsoid elliptical truncation of the form $\mathbf{a} \leq\left(\mathbf{x}-\boldsymbol{\mu}_{\mathrm{A}}\right)^{\top} \mathbf{A}\left(\mathbf{x}-\boldsymbol{\mu}_{\mathbf{A}}\right)$ on well known distributions as the multivariate normal, Student's $t$, and generalized hyperbolic distribution. On the other hand, [7] recently proposed a recursive approach that allows calculating arbitrary product moments for the normal multivariate case. Based on the latter, [8] proposes the calculation of doubly truncated moments for the normal mean-variance mixture distributions ([9]) which includes several well-known complex asymmetric multivariate distributions as the generalized hyperbolic distribution ([10]).

Unlike [8], in this paper we focus our efforts to the general class of asymmetric distributions called the multivariate elliptical selection family. This large family of distributions includes complex multivariate asymmetric versions of 
well-known elliptical distributions as the normal, Student's $t$, exponential power, hyperbolic, slash, Pearson type II, contaminated normal, among others. We go further in details for the unified skew- $t$ (SUT) distribution, a complex multivariate asymmetric heavy-tailed distribution which includes the extended skew- $t$ (EST) distribution ([11]), the skew- $t$ (ST) distribution ([12]) and naturally, as limiting cases, its analogous normal and skew-normal (SN) distributions when $\nu \rightarrow \infty$.

The rest of the paper is organized as follows. In Section 2 we present some preliminaries results, most of them being definitions of the class of distributions and its special cases of interest along the manuscript. Section 3, the addresses the moments for the doubly truncated selection elliptical distributions. Further, we establish formulas for high order moments as well as its first two moments. We present a methodology to deal with some limiting cases and a discussion when a non-truncated partition exists. In addition, we establish sufficient and necessary conditions for the existence of these truncated moments. Section 4 bases results from Section 3 to the SUT case. In Section 5, a brief numerical study is presented in order to validate the methodology. In Section 6, we present some Lemmas and Corollaries related to conditional expectations which are useful in censored modeling. An application of selection elliptical truncated moments on tail conditional expectation is presented in Section 7. Finally, the paper closes with some conclusions and direction for future research.

\section{Preliminaries}

\subsection{Selection distributions}

First, we start our exposition defining a selection distribution as in [13].

Definition 1 (selection distribution). Let $\mathbf{X}_{1} \in \mathbb{R}^{q}$ and $\mathbf{X}_{2} \in \mathbb{R}^{p}$ be two random vectors, and denote by $C$ a measurable subset of $\mathbb{R}^{q}$. We define a selection distribution as the conditional distribution of $\mathbf{X}_{2}$ given $\mathbf{X}_{1} \in C$, that is, as the distribution of $\left(\mathbf{X}_{2} \mid \mathbf{X}_{1} \in C\right)$. We say that a random vector $\mathbf{Y} \in \mathbb{R}^{p}$ has a selection distribution if $\mathbf{Y} \stackrel{d}{=}\left(\mathbf{X}_{2} \mid \mathbf{X}_{1} \in C\right)$.

We use the notation $\mathbf{Y} \sim S L C T_{p, q}$ with parameters depending on the characteristics of $\mathbf{X}_{1}, \mathbf{X}_{2}$, and $C$. Furthermore, for $\mathbf{X}_{2}$ having a probability density function (pdf) $f_{\mathbf{X}_{2}}$ say, then $\mathbf{Y}$ has a pdf $f_{\mathbf{Y}}$ given by

$$
f_{\mathbf{Y}}(\mathbf{y})=f_{\mathbf{X}_{2}}(\mathbf{y}) \frac{\mathbb{P}\left(\mathbf{X}_{1} \in C \mid \mathbf{X}_{2}=\mathbf{y}\right)}{\mathbb{P}\left(\mathbf{X}_{1} \in C\right)} .
$$

Since selection distribution depends on the subset $C \in \mathbb{R}^{q}$, particular cases are obtained. One of the most important case is when the selection subset has the form

$$
C(\mathbf{c})=\left\{\mathbf{x}_{1} \in \mathbb{R}^{q} \mid \mathbf{x}_{1}>\mathbf{c}\right\}
$$

In particular, when $\mathbf{c}=\mathbf{0}$, the distribution of $\mathbf{Y}$ is called to be a simple selection distribution.

In this work, we are mainly interested in the case where $\left(\mathbf{X}_{1}, \mathbf{X}_{2}\right)$ has a joint density following an arbitrary symmetric multivariate distribution $f_{\mathbf{X}_{1}, \mathbf{X}_{2}}$. For $\mathbf{Y} \stackrel{d}{=}\left(\mathbf{X}_{2} \mid \mathbf{X}_{1} \in C\right)$, this setting leads to a $\mathbf{Y} p$-variate random vector following a skewed version of $f$, which its pdf can be computed in a simpler manner as

$$
f_{\mathbf{Y}}(\mathbf{y})=\frac{\int_{C} f_{\mathbf{X}_{1}, \mathbf{x}_{2}}\left(\mathbf{x}_{1}, \mathbf{y}\right) \mathrm{d} \mathbf{x}_{1}}{\int_{C} f_{\mathbf{X}_{1}}\left(\mathbf{x}_{1}\right) \mathrm{d} \mathbf{x}_{1}}
$$

\subsection{Selection elliptical (SE) distributions}

A quite popular family of selection distributions arises when $\mathbf{X}_{1}$ and $\mathbf{X}_{2}$ have a joint multivariate elliptically contoured $(E C)$ distribution, as follows:

$$
\mathbf{X}=\left(\begin{array}{l}
\mathbf{X}_{1} \\
\mathbf{X}_{2}
\end{array}\right) \sim E C_{q+p}\left(\boldsymbol{\xi}=\left(\begin{array}{l}
\boldsymbol{\xi}_{1} \\
\boldsymbol{\xi}_{2}
\end{array}\right), \boldsymbol{\Omega}=\left(\begin{array}{ll}
\boldsymbol{\Omega}_{11} & \boldsymbol{\Omega}_{12} \\
\boldsymbol{\Omega}_{21} & \boldsymbol{\Omega}_{22}
\end{array}\right), h^{(q+p)}\right),
$$

where $\boldsymbol{\xi}_{1} \in \mathbb{R}^{q}$ and $\boldsymbol{\xi}_{2} \in \mathbb{R}^{p}$ are location vectors, $\boldsymbol{\Omega}_{11} \in \mathbb{R}^{q \times q}, \boldsymbol{\Omega}_{22} \in \mathbb{R}^{p \times p}$, and $\boldsymbol{\Omega}_{21} \in \mathbb{R}^{p \times q}$ are dispersion matrices, and, in addition to these parameters, $h^{(q+p)}$ is a density generator function. We denote the selection distribution resulting from (4) by $S L C T-E C_{p, q}\left(\boldsymbol{\xi}, \Omega, h^{(q+p)}, C\right)$. They typically result in skew-elliptical distributions, except for two cases: $\boldsymbol{\Omega}_{21}=\mathbf{0}_{p \times q}$ and $C=C\left(\boldsymbol{\xi}_{1}\right)$ (for more details, see [13]). Given that the elliptical family of distributions 
is closed under marginalization and conditioning, the distribution of $\mathbf{X}_{2}$ and $\left(\mathbf{X}_{1} \mid \mathbf{X}_{2}=\mathbf{x}\right)$ are also elliptical, where their respective pdfs are given by

$$
\begin{aligned}
\mathbf{X}_{2} & \sim E C_{p}\left(\boldsymbol{\xi}_{2}, \boldsymbol{\Omega}_{22}, h^{(p)}\right), \\
\mathbf{X}_{1} \mid \mathbf{X}_{2}=\mathbf{x} & \sim E C_{q}\left(\boldsymbol{\xi}_{1}+\boldsymbol{\Omega}_{12} \boldsymbol{\Omega}_{22}^{-1}\left(\mathbf{x}-\boldsymbol{\xi}_{2}\right), \boldsymbol{\Omega}_{11}-\boldsymbol{\Omega}_{12} \boldsymbol{\Omega}_{22}^{-1} \boldsymbol{\Omega}_{21}, h_{\mathbf{x}}^{(q)}\right),
\end{aligned}
$$

with induced conditional generator

$$
h_{\mathbf{x}}^{(q)}(u)=\frac{h^{(q+p)}\left(u+\delta_{2}(\mathbf{x})\right)}{h^{(p)} \delta_{2}(\mathbf{x})},
$$

with $\delta_{2}(\mathbf{x}) \triangleq\left(\mathbf{x}-\boldsymbol{\xi}_{2}\right)^{\top} \boldsymbol{\Omega}_{22}^{-1}\left(\mathbf{x}-\boldsymbol{\xi}_{2}\right)$. These last equations imply that the selection elliptical distributions are also closed under marginalization and conditioning. Furthermore, it is well-know that the SE family is closed under linear transformations. For $\mathbf{A} \in \mathbb{R}^{r \times p}$ and $\mathbf{b} \in \mathbb{R}^{r}$ being a matrix of rank $r \leq p$ and a vector, respectively, it holds that the linear transformation $\mathbf{A Y}+\mathbf{b} \stackrel{d}{=}\left(\mathbf{A X}_{2}+\mathbf{b}\right) \mid\left(\mathbf{X}_{1}>\mathbf{0}\right)$, where $\stackrel{d}{=}$ is an acronym that stands for identically distributed, and then

$$
\mathbf{A Y}+\mathbf{b} \sim S L C T-E C_{r, q}\left(\boldsymbol{\xi}=\left(\begin{array}{c}
\boldsymbol{\xi}_{1} \\
\mathbf{A} \boldsymbol{\xi}_{2}+\mathbf{b}
\end{array}\right), \boldsymbol{\Omega}=\left(\begin{array}{cc}
\boldsymbol{\Omega}_{11} & \boldsymbol{\Omega}_{12} \mathbf{A}^{\top} \\
\mathbf{A} \boldsymbol{\Omega}_{21} & \mathbf{A} \boldsymbol{\Omega}_{22} \mathbf{A}^{\top}
\end{array}\right), h^{(q+r)}\right) .
$$

Notice from Equation [3], that alternatively we can write

$$
f_{\mathbf{Y}}(\mathbf{y})=\frac{\int_{C} f_{q+p}\left(\mathbf{x}_{1}, \mathbf{y} ; \boldsymbol{\xi}, \boldsymbol{\Omega}, h^{(q+p)}\right) \mathrm{d} \mathbf{x}_{1}}{\int_{C} f_{q}\left(\mathbf{x}_{1} ; \boldsymbol{\xi}_{1}, \boldsymbol{\Omega}_{11}, h^{(q)}\right) \mathrm{d} \mathbf{x}_{1}}
$$

\subsection{Particular cases for the SE distribution}

Some particular cases, useful for our purposes, are detailed next. For further details, we refer to [13].

\section{Unified-skew elliptical (SUE) distribution}

Let $\mathbf{Y} \sim S L C T-E C_{p, q}\left(\boldsymbol{\xi}, \boldsymbol{\Omega}, h^{(q+p)}, C\right)$. $\mathbf{Y}$ is said to follow the unified skew-elliptical distribution introduced by [14] when the truncation subset $C=C(\mathbf{0})$. From [8], it follows that

$$
f_{\mathbf{Y}}(\mathbf{y})=f_{p}\left(\mathbf{y} ; \boldsymbol{\xi}_{2}, \boldsymbol{\Omega}_{22}, h^{(p)}\right) \frac{F_{q}\left(\boldsymbol{\xi}_{1}+\boldsymbol{\Omega}_{12} \boldsymbol{\Omega}_{22}^{-1}\left(\mathbf{y}-\boldsymbol{\xi}_{2}\right) ; \mathbf{0}, \boldsymbol{\Omega}_{11}-\boldsymbol{\Omega}_{12} \boldsymbol{\Omega}_{22}^{-1} \boldsymbol{\Omega}_{21}, h_{\mathbf{y}}^{(q)}\right)}{F_{q}\left(\boldsymbol{\xi}_{1} ; \boldsymbol{\Omega}_{11}, h^{(q)}\right)},
$$

where $f_{p}\left(\mathbf{y} ; \boldsymbol{\xi}_{2}, \boldsymbol{\Omega}_{22}, h^{(p)}\right)=\left|\boldsymbol{\Omega}_{22}\right|^{-1 / 2} h^{(p)}\left(\delta_{\mathbf{X}_{2}}(\mathbf{y})\right)$, and $F_{q}\left(\mathbf{z} ; \mathbf{0}, \boldsymbol{\Theta}, g^{(q)}\right)$ denote the cumulative distribution function (cdf) of the $E C_{q}\left(\mathbf{0}, \boldsymbol{\Theta}, g^{(q)}\right)$. Note that the density in (9) extends the family of skew elliptical distributions proposed by [15] (see also, [12]), which consider $q=1$ and $\xi_{1}=0$.

\section{Scale-mixture of unified-skew normal (SMSUN) distribution}

Let $W$ being a nonnegative random variable with cdf $G$. For a generator function $h^{(p+q)}(u)=$ $\int_{0}^{\infty}(2 \pi \kappa(w))^{-(p+q) / 2} e^{-u / 2 \kappa(w)} \mathrm{d} G(w)$, several skewed and thick-tailed distributions can be obtained from different specifications of the weight function $\kappa(\cdot)$ and $G$. It is said that $\mathbf{Y}$ follows a SMSUN distribution, if its probability density function (pdf) takes the general form

$$
f_{\mathbf{Y}}(\mathbf{y})=\int_{0}^{\infty} \phi_{p}\left(\mathbf{y} ; \boldsymbol{\xi}_{2}, \kappa(w) \boldsymbol{\Omega}_{22}\right) \frac{\Phi_{q}\left(\boldsymbol{\xi}_{1}+\boldsymbol{\Omega}_{12} \boldsymbol{\Omega}_{22}^{-1}\left(\mathbf{y}-\boldsymbol{\xi}_{2}\right) ; \kappa(w)\left\{\boldsymbol{\Omega}_{11}-\boldsymbol{\Omega}_{12} \boldsymbol{\Omega}_{22}^{-1} \boldsymbol{\Omega}_{21}\right\}\right)}{\Phi_{q}\left(\boldsymbol{\xi}_{1} ; \kappa(w) \boldsymbol{\Omega}_{11}\right)} \mathrm{d} G(w),
$$

where $\Phi_{r}(\cdot ; \boldsymbol{\Sigma})$ represents the cdf of a $r$-variate normal distribution with mean vector $\mathbf{0}$ and variance-covariance matrix $\boldsymbol{\Sigma}$. Here $\mathbf{Y} \mid(W=w)$ follow a unified skew-normal (SUN) distribution, where we write $\mathbf{Y} \mid(W=w) \sim$ $S U N(\boldsymbol{\xi}, \kappa(w) \boldsymbol{\Omega})$. 


\section{- Unified skew-normal (SUN) distribution}

Setting $W$ as a degenerated r.v. in $1(\mathbb{P}(W=1)=1)$ and $\kappa(w)=w$, then $h^{(p+q)}(u)=(2 \pi)^{-(p+q) / 2} e^{-u / 2}$, $u \geq 0$, for which $h^{(p)}(u)=(2 \pi)^{-p / 2} e^{-u / 2}$. Then, $\mathbf{Y}$ follow a SUN distribution, that is, $\mathbf{Y} \sim S U N_{p, q}(\boldsymbol{\xi}, \boldsymbol{\Omega})$, with pdf as

$$
f_{\mathbf{Y}}(\mathbf{y})=\phi_{p}\left(\mathbf{y} ; \boldsymbol{\xi}_{2}, \boldsymbol{\Omega}_{22}\right) \frac{\Phi_{q}\left(\boldsymbol{\xi}_{1}+\boldsymbol{\Omega}_{12} \boldsymbol{\Omega}_{22}^{-1}\left(\mathbf{y}-\boldsymbol{\xi}_{2}\right) ; \boldsymbol{\Omega}_{11}-\boldsymbol{\Omega}_{12} \boldsymbol{\Omega}_{22}^{-1} \boldsymbol{\Omega}_{21}\right)}{\Phi_{q}\left(\boldsymbol{\xi}_{1} ; \boldsymbol{\Omega}_{11}\right)} .
$$

\section{- Unified skew- $t$ (SUT) distribution}

For $W \sim G(\nu / 2, \nu / 2)$ and weight function $\kappa(w)=1 / w$, we obtain $h^{(p+q)}(u)=\frac{\Gamma((p+q+\nu) / 2) \nu^{\nu / 2}}{\Gamma(\nu / 2) \pi^{(p+q) / 2}}\{1+$ $u\}^{-(p+q+\nu) / 2}$ and hence (10) becomes

$$
f_{\mathbf{Y}}(\mathbf{y})=t_{p}\left(\mathbf{y} ; \boldsymbol{\xi}_{2}, \boldsymbol{\Omega}_{2}, \nu\right) \frac{T_{q}\left(\boldsymbol{\xi}_{1}+\boldsymbol{\Omega}_{12} \boldsymbol{\Omega}_{22}^{-1}\left(\mathbf{y}-\boldsymbol{\xi}_{2}\right) ; \frac{\nu+\delta_{2}(\mathbf{y})}{\nu+p}\left\{\boldsymbol{\Omega}_{11}-\boldsymbol{\Omega}_{12} \boldsymbol{\Omega}_{22}^{-1} \boldsymbol{\Omega}_{21}\right\}, \nu+p\right)}{T_{q}\left(\boldsymbol{\xi}_{1} ; \boldsymbol{\Omega}_{11}, \nu\right)},
$$

where $T_{r}(\cdot ; \boldsymbol{\Sigma}, \nu)$ represents the cdf of a $r$-variate Student's $t$ distribution with location vector $\mathbf{0}$, scale matrix $\boldsymbol{\Sigma}$ and degrees of freedom $\nu$. For $\mathbf{Y}$ with pdf as in (12) is said to follow a SUT distribution, which is denoted by $\mathbf{Y} \sim S U T_{p, q}(\boldsymbol{\xi}, \boldsymbol{\Omega}, \nu)$ and was introduced by [14]. It is well-know that (12) reduces to a SUN pdf (11) as $\nu \rightarrow \infty$ and to an unified skew-Cauchy (SUC) distribution, when $\nu=1$.

Furthermore, using the following parametrization:

$$
\boldsymbol{\xi}=\left(\begin{array}{c}
\boldsymbol{\tau} \\
\boldsymbol{\mu}
\end{array}\right) \quad \text { and } \quad \boldsymbol{\Omega}=\left(\begin{array}{cc}
\boldsymbol{\Psi}+\boldsymbol{\Lambda}^{\top} \boldsymbol{\Lambda} & \boldsymbol{\Omega}_{12} \\
\boldsymbol{\Omega}_{21} & \boldsymbol{\Sigma}
\end{array}\right)
$$

where $\Omega_{21}=\Sigma^{1 / 2} \boldsymbol{\Lambda}$, with $\boldsymbol{\Sigma}^{1 / 2}$ being the square root matrix of $\boldsymbol{\Sigma}$ such that $\boldsymbol{\Sigma}=\boldsymbol{\Sigma}^{1 / 2} \boldsymbol{\Sigma}^{1 / 2}$, we use the notation $\mathbf{Y} \sim S_{U} T_{p, q}(\boldsymbol{\mu}, \boldsymbol{\Sigma}, \boldsymbol{\Lambda}, \boldsymbol{\tau}, \nu, \boldsymbol{\Psi})$, to stand for a $p$-variate EST distribution with location parameter $\boldsymbol{\mu} \in \mathbb{R}^{p}$, positive-definite scale matrix $\boldsymbol{\Sigma} \in \mathbb{R}^{p \times p}$, shape matrix parameter $\boldsymbol{\lambda} \in \mathbb{R}^{p \times q}$, extension vector parameter $\boldsymbol{\tau} \in \mathbb{R}^{q}$ and positive-definite correlation matrix $\boldsymbol{\Psi} \in \mathbb{R}^{q \times q}$. The pdf $\mathbf{Y}$ is now simplified to

$$
S U T_{p, q}(\mathbf{y} ; \boldsymbol{\mu}, \boldsymbol{\Sigma}, \boldsymbol{\Lambda}, \boldsymbol{\tau}, \nu, \Psi)=t_{p}(\mathbf{y} ; \boldsymbol{\mu}, \boldsymbol{\Sigma}, \nu) \frac{T_{q}\left(\left(\boldsymbol{\tau}+\boldsymbol{\Lambda}^{\top} \boldsymbol{\Sigma}^{-1 / 2}(\mathbf{y}-\boldsymbol{\mu})\right) \nu(\mathbf{y}), \boldsymbol{\Psi} ; \nu+p\right)}{T_{q}\left(\boldsymbol{\tau} ; \boldsymbol{\Psi}+\boldsymbol{\Lambda}^{\top} \boldsymbol{\Lambda}, \nu\right)},
$$

with $\nu^{2}(\mathbf{x}) \equiv \nu_{\mathbf{X}}^{2}(\mathbf{x}) \triangleq(\nu+\operatorname{dim}(\mathbf{x})) /(\nu+\delta(\mathbf{x}))$ and $\delta(\mathbf{x})=\left(\mathbf{x}-\boldsymbol{\mu}_{\mathbf{X}}\right)^{\top} \boldsymbol{\Sigma}_{\mathbf{X}}^{-1}\left(\mathbf{x}-\boldsymbol{\mu}_{\mathbf{X}}\right)$ being the Mahalanobis distance. The pdf in (14) is equivalent to the one found in [11], with a different parametrization. Although the unified skew- $t$ distribution above is appealing from a theoretical point of view, the particular case, when $q=1$, leads to simpler but flexible enough distribution of interest for practical purposes.

\section{Extended skew- $t$ (EST) distribution}

For $q=1$, we have that $\boldsymbol{\Psi}=1, \boldsymbol{\Lambda}=\boldsymbol{\lambda}$ and $T_{q}(\mathbf{x} ; \boldsymbol{\Psi}, \nu)=T_{1}(x / \sqrt{\psi}, \nu)$, hence (14) reduces to the pdf of a EST distribution, denoted by $E S T_{p}(\mathbf{y} ; \boldsymbol{\mu}, \boldsymbol{\Sigma}, \boldsymbol{\lambda}, \tau)$, that is,

$$
\operatorname{EST}_{p}(\mathbf{y} ; \boldsymbol{\mu}, \boldsymbol{\Sigma}, \boldsymbol{\lambda}, \tau)=t_{p}(\mathbf{y} ; \boldsymbol{\mu}, \boldsymbol{\Sigma}, \nu) \frac{T_{1}\left(\left(\tau+\boldsymbol{\lambda}^{\top} \boldsymbol{\Sigma}^{-1 / 2}(\mathbf{y}-\boldsymbol{\mu})\right) \nu(\mathbf{y}) ; \nu+p\right)}{T_{1}(\tilde{\tau} ; \nu)} .
$$

with $\tilde{\tau}=\tau / \sqrt{1+\boldsymbol{\lambda}^{\top} \boldsymbol{\lambda}}$. Here, $\boldsymbol{\lambda} \in \mathbb{R}^{p}$ is a shape parameter which regulates the skewness of $\mathbf{Y}$, and $\tau \in \mathbb{R}$ is a scalar. Location and scale parameters $\boldsymbol{\mu}$ and $\boldsymbol{\Sigma}$ remains as before. Here, we write $\mathbf{Y} \sim E S T_{p}(\boldsymbol{\mu}, \boldsymbol{\Sigma}, \boldsymbol{\lambda}, \tau)$ Notice that, $S U T_{p, 1} \equiv E S T_{p}$. Besides, it is straightforward to see that

$$
E S T_{p}(\mathbf{y} ; \boldsymbol{\mu}, \boldsymbol{\Sigma}, \boldsymbol{\lambda}, \tau, \nu) \longrightarrow t_{p}(\mathbf{y} ; \boldsymbol{\mu}, \boldsymbol{\Sigma}, \nu) \text {, as } \tau \rightarrow \infty,
$$

where $t_{p}(\cdot ; \boldsymbol{\mu}, \boldsymbol{\Sigma}, \nu)$ corresponds to the pdf of a multivariate Student's $t$ distribution with location parameter $\boldsymbol{\mu}$, scale parameter $\boldsymbol{\Sigma}$ and degrees of freedom $\nu$. On the other hand, when $\tau=0$, we retrieve the skew- $t$ distribution $S T_{p}(\boldsymbol{\mu}, \boldsymbol{\Sigma}, \boldsymbol{\lambda}, \nu)$ say, which density function is given by

$$
S T_{p}(\mathbf{y} ; \boldsymbol{\mu}, \boldsymbol{\Sigma}, \boldsymbol{\lambda}, \nu)=2 t_{p}(\mathbf{y} ; \boldsymbol{\mu}, \boldsymbol{\Sigma}, \nu) T_{1}\left(\boldsymbol{\lambda}^{\top} \boldsymbol{\Sigma}^{-1 / 2}(\mathbf{y}-\boldsymbol{\mu}) \nu(\mathbf{y}) ; \nu+p\right),
$$



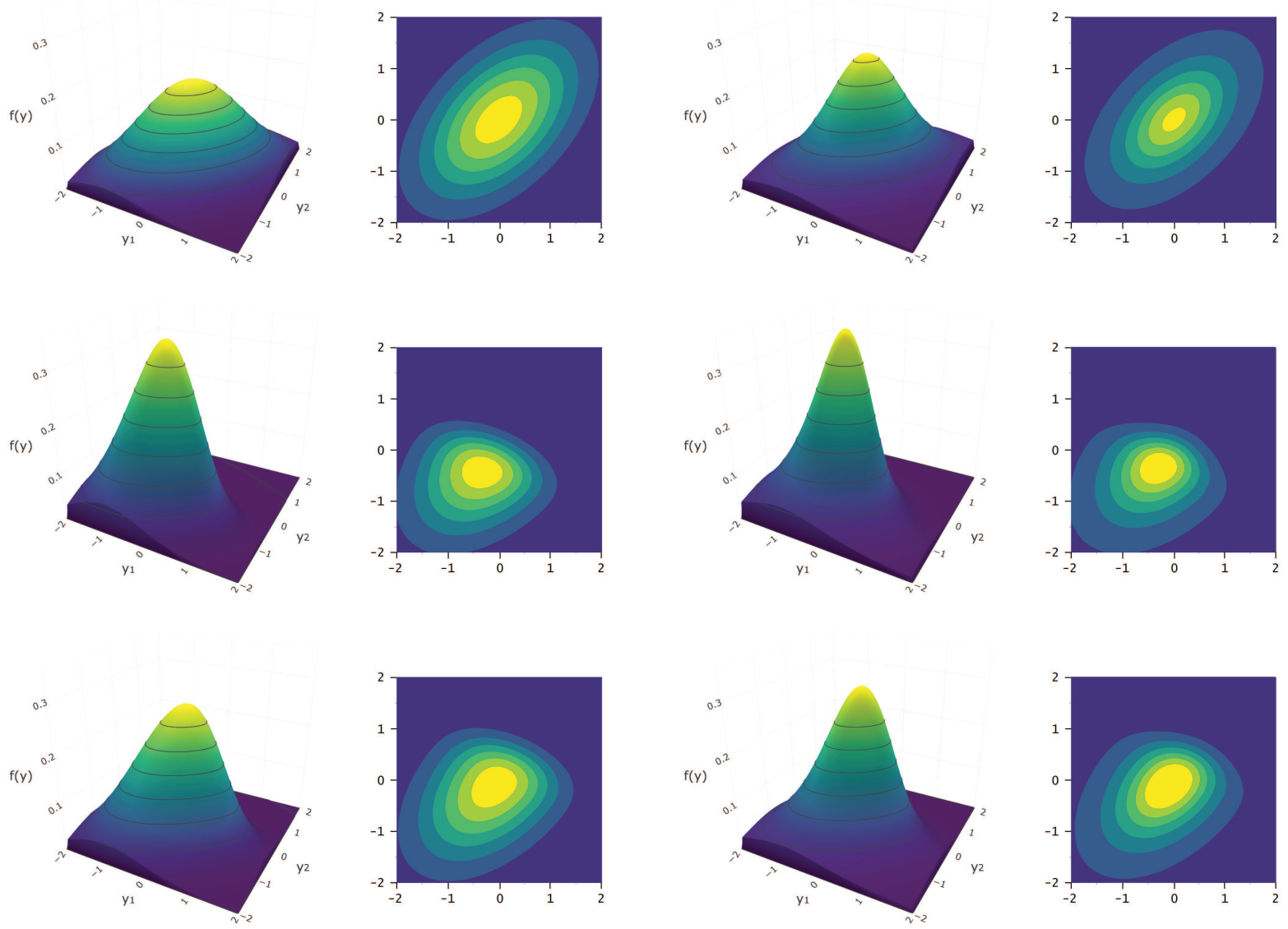

Figure 1: Densities for particular cases of a truncated SUT distribution. Normal cases at left column (normal, SN and ESN from top to bottom) and Student's- $t$ cases at right (Student's $t$, ST and EST from top to bottom).

that is, $E S T_{p}(\boldsymbol{\mu}, \boldsymbol{\Sigma}, \boldsymbol{\lambda}, 0, \nu)=S T_{p}(\boldsymbol{\mu}, \boldsymbol{\Sigma}, \boldsymbol{\lambda}, \nu)$. Further properties were studied in [11], but with a slightly different parametrization.

Six different densities for special cases of the truncated SUT distribution are shown in Figure1 Symmetrical cases normal and Student's $t$ are shown at first row $(\boldsymbol{\lambda}=\mathbf{0})$, skew cases: skew-normal $(\mathrm{SN})$ and ST at second row $(\tau=0)$ and extended skew cases: extended skew-normal $(\mathrm{ESN})$ and EST at the third row. Location vector $\boldsymbol{\mu}$ and scale matrix $\boldsymbol{\Sigma}$ remains fixed for all cases.

\section{- Others unified skewed distributions}

Others unified members are given by different combinations of the weight function $\kappa(W)$ and the mixture cdf $G$. For instance, we obtain an unified skew-slash distribution when $\kappa(w)=1 / w$ and $W \sim \operatorname{Beta}(\nu, 1)$; an unified skew-contaminated-normal distribution when $\kappa(W)=1 / W$ and $W$ is a discrete r.v. with probability mass function (pmf) $g(w ; \nu, \gamma)=\nu \mathbb{I}_{\{w=\gamma\}}+(1-\nu) \mathbb{I}_{\{w=1\}}$, with $\mathbb{I}$ being the identity function. Besides, [15] mentions some other distributions as the skew-logistic, skew-stable, skew-exponential power, skew-Pearson type II and finite mixture of skew-normal distribution. It is worth mentioning that even though [15] works with a subclass of the SMSUN, when $q=1$ and $\xi_{1}=0$, unified versions of these are readily computed by considering the same respective weight function $\kappa(\cdot)$ and mixture distribution $G$.

\section{On moments of the doubly truncated selection elliptical distribution}

Let $\mathbf{Y} \sim S L C T-E C_{p, q}\left(\boldsymbol{\xi}, \boldsymbol{\Omega}, h^{(q+p)}, C\right)$ with pdf as defined in $(8)$ and let also $\mathbb{A}$ be a Borel set in $\mathbb{R}^{p}$. We say that a random vector $\mathbf{W}$ has a truncated selection elliptical (TSE) distribution on $\mathbb{A}$ when $\mathbf{W} \stackrel{d}{=} \mathbf{Y} \mid(\mathbf{Y} \in \mathbb{A})$. In this case, 
the pdf of $\mathbf{W}$ is given by

$$
f_{\mathbf{W}}(\mathbf{w})=\frac{f_{\mathbf{Y}}(\mathbf{w})}{P(\mathbf{Y} \in \mathbb{A})} \mathbf{1}_{\mathbb{A}}(\mathbf{w})
$$

where $\mathbf{1}_{\mathbb{A}}$ is the indicator function of the set $\mathbb{A}$. We use the notation $\mathbf{W} \sim T S L C T-E C_{p, q}\left(\boldsymbol{\xi}, \boldsymbol{\Omega}, h^{(q+p)}, C ; \mathbb{A}\right)$. If $\mathbb{A}$ has the form

$$
\mathbb{A}=\left\{\left(y_{1}, \ldots, y_{p}\right) \in \mathbb{R}^{p}: a_{1} \leq y_{1} \leq b_{1}, \ldots, a_{p} \leq y_{p} \leq b_{p}\right\}=\left\{\mathbf{y} \in \mathbb{R}^{p}: \mathbf{a} \leq \mathbf{y} \leq \mathbf{b}\right\},
$$

we say that the distribution of $\mathbf{W}$ is doubly truncated distribution and we use the notation $\{\mathbf{Y} \in \mathbb{A}\}=\{\mathbf{a} \leq \mathbf{Y} \leq \mathbf{b}\}$, where $\mathbf{a}=\left(a_{1}, \ldots, a_{p}\right)^{\top}$ and $\mathbf{b}=\left(b_{1}, \ldots, b_{p}\right)^{\top}$, where $a_{i}$ and $b_{i}$ values may be infinite, by convention. Analogously we define $\{\mathbf{Y} \geq \mathbf{a}\}$ and $\{\mathbf{Y} \leq \mathbf{b}\}$. Thus, we say that the distribution of $\mathbf{W}$ is truncated from below and truncated from above, respectively. For convenience, we also use the notation $\mathbf{W} \sim T S L C T-E C_{p, q}\left(\boldsymbol{\xi}, \boldsymbol{\Omega}, h^{(q+p)}, C ;(\mathbf{a}, \mathbf{b})\right)$ with the last parameter indicating the truncation interval. Analogously, we do denote $T E C_{p}\left(\boldsymbol{\xi}, \boldsymbol{\Omega}, h^{(p)} ;(\mathbf{a}, \mathbf{b})\right)$ to refer to a $p$-variate (doubly) truncated elliptical (TE) distribution on $(\mathbf{a}, \mathbf{b}) \in \mathbb{R}^{p}$. Some characterizations of the doubly TE have been recently discussed in [16].

\subsection{Moments of a TSE distribution}

For two $p$-dimensional vectors $\mathbf{y}=\left(y_{1}, \ldots, y_{p}\right)^{\top}$ and $\mathbf{k}=\left(k_{1}, \ldots, k_{p}\right)^{\top}$, let $\mathbf{y}^{\mathbf{k}}$ stand for $\left(y_{1}^{k_{1}}, \ldots, y_{p}^{k_{p}}\right)$, that is, we use a pointwise notation. Next, we present a formulation to compute arbitrary product moments of a TSLCT-EC distribution.

Theorem 1 (moments of a TSE). Let $\mathbf{X} \sim E C_{q+p}\left(\boldsymbol{\xi}, \boldsymbol{\Omega}, h^{(q+p)}\right)$ as defined in (44). Let $C$ be a truncation subset of the form $C(\mathbf{c}, \mathbf{d})=\left\{\mathbf{x}_{1} \in \mathbb{R}^{q} \mid \mathbf{c} \leq \mathbf{x}_{1} \leq \mathbf{d}\right\}$. For $\mathbf{Y} \sim S L C T-E C_{p, q}\left(\boldsymbol{\xi}, \boldsymbol{\Omega}, h^{(q+p)}, C(\mathbf{c}, \mathbf{d})\right)$, then $\mathbb{E}\left[\mathbf{Y}^{\mathbf{k}}\right]=$ $\mathbb{E}\left[Y_{1}^{k_{1}} Y_{2}^{k_{2}} \ldots Y_{p}^{k_{p}}\right]$ can be computed as

$$
\mathbb{E}\left[\mathbf{Y}^{\mathbf{k}} \mid \mathbf{a} \leq \mathbf{Y} \leq \mathbf{b}\right]=\mathbb{E}\left[\mathbf{X}^{\kappa} \mid \boldsymbol{\alpha} \leq \mathbf{X} \leq \boldsymbol{\beta}\right],
$$

with $\boldsymbol{\kappa}=\left(\mathbf{0}_{q}^{\top}, \mathbf{k}^{\top}\right)^{\top}, \boldsymbol{\alpha}=\left(\mathbf{c}^{\top}, \mathbf{a}^{\top}\right)^{\top}$ and $\boldsymbol{\beta}=\left(\mathbf{d}^{\top}, \mathbf{b}^{\top}\right)^{\top}$, where $\mathbf{k}=\left(k_{1}, k_{2}, \ldots, k_{p}\right)^{\top}$, with $k_{i} \in \mathbb{N}$, for $i=1, \ldots, p$.

Proof. Since $\mathbf{Y} \stackrel{d}{=} \mathbf{X}_{2} \mid\left(\mathbf{c} \leq \mathbf{X}_{1} \leq \mathbf{d}\right)$, the proof is direct by noting that

$$
\begin{aligned}
\mathbf{Y} \mid(\mathbf{a} \leq \mathbf{Y} \leq \mathbf{b}) & \stackrel{d}{=} \mathbf{X}_{2} \mid\left(\mathbf{c} \leq \mathbf{X}_{1} \leq \mathbf{d} \cap \mathbf{a} \leq \mathbf{X}_{2} \leq \mathbf{b}\right) \\
& \stackrel{d}{=} \mathbf{X}_{2} \mid(\boldsymbol{\alpha} \leq \mathbf{X} \leq \boldsymbol{\beta})
\end{aligned}
$$

Corollary 1 (first two moments of a TSE). Under the same conditions of Theorem 1, let $\mathbf{m}=\mathbb{E}[\mathbf{X} \mid \boldsymbol{\alpha} \leq \mathbf{X} \leq \boldsymbol{\beta}]$ and $\mathbf{M}=\mathbb{E}\left[\mathbf{X X}^{\top} \mid \boldsymbol{\alpha} \leq \mathbf{X} \leq \boldsymbol{\beta}\right]$, both partitioned as

$$
\mathbf{m}=\left(\begin{array}{l}
\mathbf{m}_{1} \\
\mathbf{m}_{2}
\end{array}\right) \quad \text { and } \quad \mathbf{M}=\left(\begin{array}{ll}
\mathbf{M}_{11} & \mathbf{M}_{12} \\
\mathbf{M}_{21} & \mathbf{M}_{22}
\end{array}\right),
$$

respectively. Then, the first two moments of $\mathbf{Y} \mid(\mathbf{a} \leq \mathbf{Y} \leq \mathbf{b})$ are given by

$$
\begin{aligned}
\mathbb{E}[\mathbf{Y} \mid \mathbf{a} \leq \mathbf{Y} \leq \mathbf{b}] & =\mathbf{m}_{2}, \\
\mathbb{E}\left[\mathbf{Y} \mathbf{Y}^{\top} \mid \mathbf{a} \leq \mathbf{Y} \leq \mathbf{b}\right] & =\mathbf{M}_{22},
\end{aligned}
$$

where $\mathbf{m}_{2} \in \mathbb{R}^{p}$ and $\mathbf{M}_{22} \in \mathbb{R}^{p \times p}$.

For the particular truncation subset $C(\mathbf{c})$ as in (2), Theorem 1 and Corollary 1 hold considering $\boldsymbol{\alpha}=\left(\mathbf{c}^{\top}, \mathbf{a}^{\top}\right)^{\top}$ and $\boldsymbol{\beta}=\left(\boldsymbol{\infty}^{\top}, \mathbf{b}^{\top}\right)^{\top}$. Notice that, Theorem 1 and Corollary 1 state that we are able to compute any arbitrary moment of $\mathbf{Y} \mid(\mathbf{a} \leq \mathbf{Y} \leq \mathbf{b})$, that is, a TSE distribution just using an unique corresponding moment of a doubly TE distribution $\mathbf{X} \mid(\boldsymbol{\alpha} \leq \mathbf{X} \leq \boldsymbol{\beta})$.

This is highly convenient since doubly truncated moments for some members of the elliptical family of distributions are already available in the literature and statistical softwares. In particular for the truncated multivariate normal and Student's-t we have the R packages TTmoment, tmvtnorm and MomTrunc. 


\subsection{Dealing with limiting and extreme cases}

Consider $\mathbf{X} \sim E C_{q+p}\left(\boldsymbol{\xi}, \boldsymbol{\Omega}, h^{(q+p)}\right)$ and $\mathbf{Y} \sim S L C T-E C_{p, q}\left(\boldsymbol{\xi}, \boldsymbol{\Omega}, h^{(q+p)}, C\right)$ as in Theorem 1 with truncation subset $C=C(\mathbf{0})$. As $\boldsymbol{\xi}_{1} \rightarrow \infty$, we have that $\mathbb{P}\left(\mathbf{X}_{1} \geq \mathbf{0}\right) \rightarrow 1$. Besides, as $\boldsymbol{\xi}_{1} \rightarrow-\infty$, we have that $\mathbb{P}\left(\mathbf{X}_{1} \geq \mathbf{0}\right) \rightarrow 0$ and consequently $\mathbb{P}(\mathbf{a} \leq \mathbf{Y} \leq \mathbf{b})=\mathbb{P}(\boldsymbol{\alpha} \leq \mathbf{X} \leq \boldsymbol{\beta}) / \mathbb{P}\left(\mathbf{X}_{1} \geq \mathbf{0}\right) \rightarrow \infty$. Thus, for $\boldsymbol{\xi}_{1}$ containing high negative values small enough, sometimes we are not able to compute $\mathbb{E}\left[\mathbf{Y}^{\mathbf{k}}\right]$ due to computation precision, mainly when we work with distributions with lighter tails densities. For instance, for a normal univariate case, $\Phi_{1}\left(\xi_{1}\right)=0$ for $\xi_{1} \leq-38$ in $\mathrm{R}$ software. The next proposition helps us to circumvent this problem.

Proposition 1 (limiting case of a SE). As $\boldsymbol{\xi}_{1} \rightarrow-\infty$, i.e., $\xi_{1 i} \rightarrow-\infty, i=1, \ldots, q$, then

$$
S L C T-E C_{p, q}\left(\boldsymbol{\xi}, \boldsymbol{\Omega}, h^{(q+p)}, C(\mathbf{0})\right) \longrightarrow E C_{p}\left(\boldsymbol{\xi}_{2}-\boldsymbol{\Omega}_{21} \boldsymbol{\Omega}_{11}^{-1} \boldsymbol{\xi}_{1}, \boldsymbol{\Omega}_{22}-\boldsymbol{\Omega}_{21} \boldsymbol{\Omega}_{11}^{-1} \boldsymbol{\Omega}_{12}, h_{\mathbf{0}}^{(p)}\right) .
$$

Proof. Let $\mathbf{X}=\left(\mathbf{X}_{1}^{\top}, \mathbf{X}_{2}^{\top}\right)^{\top} \sim E C_{q+p}\left(\boldsymbol{\xi}, \boldsymbol{\Omega}, h^{(q+p)}\right)$ and $\mathbf{Y} \sim T S L C T-E C_{p, q}\left(\boldsymbol{\xi}, \boldsymbol{\Omega}, h^{(q+p)}, C(\mathbf{0}) ;(\mathbf{a}, \mathbf{b})\right)$. As $\boldsymbol{\xi}_{1} \rightarrow-\infty$, we have that $\mathbb{P}\left(\mathbf{X}_{1} \geq \mathbf{0}\right) \rightarrow 0, \mathbb{E}\left[\mathbf{X}_{1} \mid \mathbf{X}_{1} \geq \mathbf{0}\right] \rightarrow \mathbf{0}$ and $\operatorname{var}\left[\mathbf{X}_{1} \mid \mathbf{X}_{1} \geq \mathbf{0}\right] \rightarrow \mathbf{0}$, hence $\mathbf{X}_{1} \mid \mathbf{X}_{1} \geq \mathbf{0}$ becomes degenerated on $\mathbf{0}$. From Definition $1, \mathbf{Y} \stackrel{d}{\longrightarrow}\left(\mathbf{X}_{2} \mid \mathbf{X}_{1}=\mathbf{0}\right)$, and by the conditional distribution in Equation (6), it is straightforward to show that $\mathbf{X}_{2} \mid \mathbf{X}_{1} \sim E C_{p}\left(\boldsymbol{\xi}_{2}+\boldsymbol{\Omega}_{21} \boldsymbol{\Omega}_{11}^{-1}\left(\mathbf{X}_{1}-\boldsymbol{\xi}_{1}\right), \boldsymbol{\Omega}_{22}-\boldsymbol{\Omega}_{21} \boldsymbol{\Omega}_{11}^{-1} \boldsymbol{\Omega}_{12}, h_{\mathbf{X}_{1}}^{(p)}\right)$. Evaluating $\mathbf{X}_{1}=\mathbf{0}$ we achieve (21) concluding the proof.

\subsection{Approximating the mean and variance-covariance of a TE distribution for extreme cases}

While using the relation (19) and (20), we may face numerical problems trying to compute $\mathbf{m}=\mathbb{E}[\mathbf{X} \mid \boldsymbol{\alpha} \leq \mathbf{X} \leq \boldsymbol{\beta}]$ and $\mathbf{M}=\mathbb{E}\left[\mathbf{X X}^{\top} \mid \boldsymbol{\alpha} \leq \mathbf{X} \leq \boldsymbol{\beta}\right]$ for extreme settings of $\boldsymbol{\xi}$ and $\boldsymbol{\Omega}$. Usually, it occurs when $\mathbb{P}(\boldsymbol{\alpha} \leq \mathbf{X} \leq \boldsymbol{\beta}) \approx 0$ because the probability density is far from the integration region $(\boldsymbol{\alpha}, \boldsymbol{\beta})$. It is worth mentioning that, for these cases, it is not even possible to estimate the moments generating Monte Carlo (MC) samples via rejection sample due to the high rejection ratio when subsetting to a small integration region. Other methods as Gibbs sampling are preferable under this situation.

Hence, we present correction method in order to approximate the mean and the variance-covariance of a multivariate TE distribution even when the numerical precision of the software is a limitation.

\subsubsection{Dealing with out-of-bounds limits}

Consider $\mathbf{X} \sim E C_{r}\left(\boldsymbol{\xi}, \boldsymbol{\Omega}, h^{(r)}\right)$ to be partitioned as $\mathbf{X}=\left(\mathbf{X}_{1}^{T}, \mathbf{X}_{2}^{\top}\right)^{\top}$ such that $\operatorname{dim}\left(\mathbf{X}_{1}\right)=r_{1}, \operatorname{dim}\left(\mathbf{X}_{2}\right)=r_{2}$, where $r_{1}+r_{2}=r$. Also, consider $\boldsymbol{\xi}, \boldsymbol{\Omega}, \boldsymbol{\alpha}=\left(\boldsymbol{\alpha}_{1}^{\top}, \boldsymbol{\alpha}_{2}^{\top}\right)^{\top}$ and $\boldsymbol{\beta}=\left(\boldsymbol{\beta}_{1}^{\top}, \boldsymbol{\beta}_{2}^{\top}\right)^{\top}$ partitioned as before. Suppose that we are not able to compute $\mathbb{E}\left[\mathbf{X}^{\kappa} \mid \boldsymbol{\alpha} \leq \mathbf{X} \leq \boldsymbol{\beta}\right]$, because there exists a partition $\mathbf{X}_{2}$ of $\mathbf{X}$ of dimension $r_{2}$ that is out-ofbounds, that is $P\left(\boldsymbol{\alpha}_{2} \leq \mathbf{X}_{2} \leq \boldsymbol{\beta}_{2}\right) \approx 0$. Notice that this happens because $\mathbb{P}(\boldsymbol{\alpha} \leq \mathbf{X} \leq \boldsymbol{\beta}) \leq P\left(\boldsymbol{\alpha}_{2} \leq \mathbf{X}_{2} \leq \boldsymbol{\beta}_{2}\right) \approx 0$. Besides, we suppose that $P\left(\boldsymbol{\alpha}_{1} \leq \mathbf{X}_{1} \leq \boldsymbol{\beta}_{1}\right)>0$. Since the limits of $\mathbf{X}_{2}$ are out-of-bounds (and $\boldsymbol{\alpha}_{2}<\boldsymbol{\beta}_{2}$ ), we have two possible cases: $\boldsymbol{\beta}_{2} \rightarrow-\infty$ or $\boldsymbol{\alpha}_{2} \rightarrow \infty$. For convenience, let $\boldsymbol{\mu}_{2}=\mathbb{E}\left[\mathbf{X}_{2} \mid \boldsymbol{\alpha}_{2} \leq \mathbf{X}_{2} \leq \boldsymbol{\beta}_{2}\right]$ and $\boldsymbol{\Sigma}_{22}=\operatorname{cov}\left[\mathbf{X}_{2} \mid \boldsymbol{\alpha}_{2} \leq \mathbf{X}_{2} \leq \boldsymbol{\beta}_{2}\right]$. For the first case, as $\boldsymbol{\beta}_{2} \rightarrow-\infty$, we have that $\boldsymbol{\mu}_{2} \rightarrow \boldsymbol{\beta}_{2}$ and $\boldsymbol{\Sigma}_{22} \rightarrow \mathbf{0}_{r_{2} \times r_{2}}$. Analogously, we have that $\boldsymbol{\mu}_{2} \rightarrow \boldsymbol{\alpha}_{2}$ and $\boldsymbol{\Sigma}_{22} \rightarrow \mathbf{0}_{r_{2} \times r_{2}}$ as $\boldsymbol{\alpha}_{2} \rightarrow \infty$. Hence, $\mathbf{X}_{2} \mid\left(\boldsymbol{\alpha}_{2} \leq \mathbf{X}_{2} \leq \boldsymbol{\beta}_{2}\right)$ is degenerated on $\boldsymbol{\mu}_{2}$ and then $\mathbf{X}_{1.2} \stackrel{d}{=} \mathbf{X}_{1} \mid\left(\mathbf{X}_{2}=\boldsymbol{\mu}_{2}\right) \sim E C_{r_{1}}\left(\boldsymbol{\xi}_{1}+\boldsymbol{\Omega}_{12} \boldsymbol{\Omega}_{22}^{-1}\left(\boldsymbol{\mu}_{2}-\boldsymbol{\xi}_{2}\right), \boldsymbol{\Omega}_{11}-\boldsymbol{\Omega}_{12} \boldsymbol{\Omega}_{22}^{-1} \boldsymbol{\Omega}_{21}, h_{\boldsymbol{\mu}_{2}}^{\left(r_{1}\right)}\right)$. Given that $\operatorname{cov}\left[\mathbb{E}\left[\mathbf{X}_{1} \mid \mathbf{X}_{2}\right]\right]=\mathbf{0}$ and $\operatorname{cov}\left[\mathbb{E}\left[\mathbf{X}_{1} \mid \mathbf{X}_{2}\right], \mathbf{X}_{2}\right]=\mathbf{0}$, it follows that

$$
\mathbb{E}[\mathbf{X} \mid \boldsymbol{\alpha} \leq \mathbf{X} \leq \boldsymbol{\beta}]=\left[\begin{array}{c}
\boldsymbol{\mu}_{1.2} \\
\boldsymbol{\mu}_{2}
\end{array}\right] \quad \text { and } \quad \operatorname{cov}[\mathbf{X} \mid \boldsymbol{\alpha} \leq \mathbf{X} \leq \boldsymbol{\beta}]=\left[\begin{array}{cc}
\boldsymbol{\Sigma}_{11.2} & \mathbf{0}_{r_{1} \times r_{2}} \\
\mathbf{0}_{r_{2} \times r_{1}} & \mathbf{0}_{r_{2} \times r_{2}}
\end{array}\right],
$$

with $\boldsymbol{\mu}_{1.2}=\mathbb{E}\left[\mathbf{X}_{1.2} \mid \boldsymbol{\alpha}_{1} \leq \mathbf{X}_{1.2} \leq \boldsymbol{\beta}_{1}\right]$ and $\boldsymbol{\Sigma}_{11.2}=\operatorname{cov}\left[\mathbf{X}_{1.2} \mid \boldsymbol{\alpha}_{1} \leq \mathbf{X}_{1.2} \leq \boldsymbol{\beta}_{1}\right]$ being the mean and variance-covariance matrix of a $r_{1}$-variate TE distribution.

In the event that there are double infinite limits, we can part the vector as well, in order to avoid unnecessary calculation of these integrals.

\subsubsection{Dealing with double infinite limits}

Now, consider $\mathbf{X}=\left(\mathbf{X}_{1}^{\top}, \mathbf{X}_{2}^{\top}\right)^{\top}$ to be partitioned such that the upper and lower truncation limits associated with $\mathbf{X}_{1}$ are both infinite, but at least one of the truncation limits associated with $\mathbf{X}_{2}$ is finite. Then $r_{1}$ be the number of pairs in $(\boldsymbol{\alpha}, \boldsymbol{\beta})$ that are both infinite, that is, $\operatorname{dim}\left(\mathbf{X}_{1}\right)=r_{1}$ and $\operatorname{dim}\left(\mathbf{X}_{2}\right)=r_{2}$, by complement. Since $\boldsymbol{\alpha}_{1}=-\infty$ and $\boldsymbol{\beta}_{1}=\boldsymbol{\infty}$, it follows that $\mathbf{X}_{2} \mid(\boldsymbol{\alpha} \leq \mathbf{X} \leq \boldsymbol{\beta}) \sim T E C_{r_{2}}\left(\boldsymbol{\xi}_{2}, \boldsymbol{\Omega}_{22}, h^{\left(r_{2}\right)} ;\left[\boldsymbol{\alpha}_{2}, \boldsymbol{\beta}_{2}\right]\right)$ and $\mathbf{X}_{1} \mid \mathbf{X}_{2} \sim E C_{r_{1}}\left(\boldsymbol{\xi}_{1}+\right.$ 
$\left.\boldsymbol{\Omega}_{12} \boldsymbol{\Omega}_{22}^{-1}\left(\mathbf{X}_{2}-\boldsymbol{\xi}_{2}\right), \boldsymbol{\Omega}_{11}-\boldsymbol{\Omega}_{12} \boldsymbol{\Omega}_{22}^{-1} \boldsymbol{\Omega}_{21}, h_{\mathbf{X}_{2}}^{\left(r_{1}\right)}\right)$. Let $\boldsymbol{\mu}_{2}=\mathbb{E}\left[\mathbf{X}_{2} \mid \boldsymbol{\alpha}_{2} \leq \mathbf{X}_{2} \leq \boldsymbol{\beta}_{2}\right]$ and $\boldsymbol{\Sigma}_{22}=\operatorname{cov}\left[\mathbf{X}_{2} \mid \boldsymbol{\alpha}_{2} \leq\right.$ $\left.\mathbf{X}_{2} \leq \boldsymbol{\beta}_{2}\right]$. Hence, it follows that $\mathbb{E}[\mathbf{X} \mid \boldsymbol{\alpha} \leq \mathbf{X} \leq \boldsymbol{\beta}]=\mathbb{E}\left[\mathbb{E}\left[\mathbf{X}_{1} \mid \mathbf{X}_{2}\right] \mid \boldsymbol{\alpha}_{2} \leq \mathbf{X}_{2} \leq \boldsymbol{\beta}_{2}\right]$, that is

$$
\begin{aligned}
\mathbb{E}[\mathbf{X} \mid \boldsymbol{\alpha} \leq \mathbf{X} \leq \boldsymbol{\beta}] & =\mathbb{E}\left[\left(\begin{array}{c}
\boldsymbol{\xi}_{1}+\boldsymbol{\Omega}_{12} \boldsymbol{\Omega}_{22}^{-1}\left(\mathbf{X}_{2}-\boldsymbol{\xi}_{2}\right) \\
\mathbf{X}_{2}
\end{array}\right) \mid \boldsymbol{\alpha}_{2} \leq \mathbf{X}_{2} \leq \boldsymbol{\beta}_{2}\right] \\
& =\left[\begin{array}{c}
\boldsymbol{\xi}_{1}+\boldsymbol{\Omega}_{12} \boldsymbol{\Omega}_{22}^{-1}\left(\boldsymbol{\mu}_{2}-\boldsymbol{\xi}_{2}\right) \\
\boldsymbol{\mu}_{2}
\end{array}\right] .
\end{aligned}
$$

On the other hand, we have that $\operatorname{cov}\left[\mathbf{X}_{2}, \mathbb{E}\left[\mathbf{X}_{1} \mid \mathbf{X}_{2}\right]\right]=\operatorname{cov}\left[\mathbf{X}_{2}, \mathbf{X}_{2} \boldsymbol{\Omega}_{22}^{-1} \boldsymbol{\Omega}_{21}\right]=\boldsymbol{\Sigma}_{22} \boldsymbol{\Omega}_{22}^{-1} \boldsymbol{\Omega}_{21}, \operatorname{cov}\left[\mathbb{E}\left[\mathbf{X}_{1} \mid \mathbf{X}_{2}\right]\right]=$ $\boldsymbol{\Omega}_{12} \boldsymbol{\Omega}_{22}^{-1} \boldsymbol{\Sigma}_{22} \boldsymbol{\Omega}_{22}^{-1} \boldsymbol{\Omega}_{21}$ and $\mathbb{E}\left[\operatorname{cov}\left[\mathbf{X}_{1} \mid \mathbf{X}_{2}\right]\right]=\omega_{1.2}\left(\boldsymbol{\Omega}_{11}-\boldsymbol{\Omega}_{12} \boldsymbol{\Omega}_{22}^{-1} \boldsymbol{\Omega}_{21}\right)$, with $\omega_{1.2}$ being a constant depending of the conditional generating function $h_{\mathbf{X}_{2}}^{\left(r_{1}\right)}$. Finally,

$$
\operatorname{cov}[\mathbf{X} \mid \boldsymbol{\alpha} \leq \mathbf{X} \leq \boldsymbol{\beta}]=\left[\begin{array}{cc}
\omega_{1.2} \boldsymbol{\Omega}_{11}-\boldsymbol{\Omega}_{12} \boldsymbol{\Omega}_{22}^{-1}\left(\omega_{1.2} \mathbf{I}_{p_{2}}-\boldsymbol{\Sigma}_{22} \boldsymbol{\Omega}_{22}^{-1}\right) \boldsymbol{\Omega}_{21} & \boldsymbol{\Omega}_{12} \boldsymbol{\Omega}_{22}^{-1} \boldsymbol{\Sigma}_{22} \\
\boldsymbol{\Sigma}_{22} \boldsymbol{\Omega}_{22}^{-1} \boldsymbol{\Omega}_{21} & \boldsymbol{\Sigma}_{22}
\end{array}\right],
$$

where $\boldsymbol{\mu}_{2}$ and $\boldsymbol{\Sigma}_{22}$ are the mean vector and variance-covariance matrix of a TE distribution, so we can use (19) and (20) as well.

Remark 1. Note that $\mathbf{X}_{1} \mid(\boldsymbol{\alpha} \leq \mathbf{X} \leq \boldsymbol{\beta})$ does not follow a non-truncated elliptical distribution, that is, $\mathbf{X}_{1} \mid(\boldsymbol{\alpha} \leq$ $\mathbf{X} \leq \boldsymbol{\beta}) \nsim E C_{r_{1}}\left(\boldsymbol{\xi}_{1}, \boldsymbol{\Omega}_{11}, h^{\left(r_{1}\right)}\right)$ even though $-\infty \leq \mathbf{X}_{1} \leq \boldsymbol{\infty}$. This occurs due to $\mathbf{X}_{1}\left|(\boldsymbol{\alpha} \leq \mathbf{X} \leq \boldsymbol{\beta})=\mathbf{X}_{1}\right|$ $\left(\boldsymbol{\alpha}_{2} \leq \mathbf{X}_{2} \leq \boldsymbol{\beta}_{2}\right)$. In general, the marginal distributions of a TE distribution are not TE, however this holds for $\mathbf{X}_{2}$ due to the particular case $\boldsymbol{\alpha}_{1}=-\infty$ and $\boldsymbol{\beta}_{1}=\infty$.

\section{Particular cases}

Notice that the constant $\omega_{1.2}$ will vary depending of the elliptical distribution we are using. For instance, if $\mathbf{X} \sim$ $t_{r_{1}+r_{2}}(\boldsymbol{\xi}, \boldsymbol{\Omega}, \nu)$ then it follows that $\mathbf{X}_{2} \sim t_{r_{2}}\left(\boldsymbol{\xi}_{2}, \boldsymbol{\Omega}_{22}, \nu\right)$ and $\mathbf{X}_{1} \mid \mathbf{X}_{2} \sim t_{r_{1}}\left(\boldsymbol{\xi}_{1}+\boldsymbol{\Omega}_{12} \boldsymbol{\Omega}_{22}^{-1}\left(\mathbf{X}_{2}-\boldsymbol{\xi}_{2}\right),\left(\boldsymbol{\Omega}_{11}-\right.\right.$ $\left.\left.\boldsymbol{\Omega}_{12} \boldsymbol{\Omega}_{22}^{-1} \boldsymbol{\Omega}_{21}\right) / \nu^{2}\left(\mathbf{X}_{2}\right), \nu+r_{2}\right)$. In this case, it takes the form $\omega_{1.2}=\mathbb{E}\left[\left(\nu+r_{2}\right) /\left(\nu+r_{2}-2\right) \nu^{2}\left(\mathbf{X}_{2}\right) \mid \boldsymbol{\alpha}_{2} \leq \mathbf{X}_{2} \leq \boldsymbol{\beta}_{2}\right]$, which is given by

$$
\begin{aligned}
\omega_{1.2} & =\mathbb{E}\left[\frac{\nu+\delta\left(\mathbf{X}_{2}\right)}{\nu+r_{2}-2} \mid \boldsymbol{\alpha}_{2} \leq \mathbf{X}_{2} \leq \boldsymbol{\beta}_{2}\right] \\
& =\left(\frac{\nu}{\nu-2}\right) \frac{L_{r_{2}}\left(\boldsymbol{\alpha}_{2}, \boldsymbol{\beta}_{2} ; \boldsymbol{\xi}_{2}, \nu \boldsymbol{\Omega}_{22} /(\nu-2), \nu-2\right)}{L_{r_{2}}\left(\boldsymbol{\alpha}_{2}, \boldsymbol{\beta}_{2} ; \boldsymbol{\xi}_{2}, \boldsymbol{\Omega}_{22}, \nu\right)}
\end{aligned}
$$

where $L_{r}(\boldsymbol{\alpha}, \boldsymbol{\beta} ; \boldsymbol{\xi}, \boldsymbol{\Omega}, \nu)$ denotes the integral

$$
L_{r}(\boldsymbol{\alpha}, \boldsymbol{\beta} ; \boldsymbol{\xi}, \boldsymbol{\Omega}, \nu)=\int_{\boldsymbol{\alpha}}^{\boldsymbol{\beta}} t_{r}(\mathbf{y} ; \boldsymbol{\xi}, \boldsymbol{\Omega}, \nu) \mathrm{d} \mathbf{y},
$$

that is, $L_{r}(\boldsymbol{\alpha}, \boldsymbol{\beta} ; \boldsymbol{\xi}, \boldsymbol{\Omega}, \nu)=\mathbb{P}(\boldsymbol{\alpha} \leq \mathbf{Y} \leq \boldsymbol{\beta})$ for $\mathbf{Y} \sim t_{r}(\boldsymbol{\xi}, \boldsymbol{\Omega}, \nu)$. Probabilities in (25) are involved in the calculation of $\boldsymbol{\mu}_{2}$ and $\boldsymbol{\Sigma}_{22}$ so they are recycled. For the normal case, it is straightforward to see that $\omega_{1.2}=1$, by taking $\nu \rightarrow \infty$.

As can be seen, we can use equations (23) and (24) to deal with double infinite limits, where the truncated moments are computed only over a $r_{2}$-variate partition, avoiding some unnecessary integrals and saving significant computational effort. On the other hand, expression (22) let us to approximate the mean and the variance-covariance matrix for cases where the computational precision is a limitation.

\subsection{Existence of the moments of a TE and TSE distribution}

It is well know that for some members of EC family of distributions, their moments do not exist, however, this could be different depending of the truncation limits.

Let $\mathbf{X} \sim E C_{r}\left(\boldsymbol{\xi}, \boldsymbol{\Omega}, h^{(r)}\right)$ be partitioned as in Subsection 3.3.2 with $r_{1}$ being the number of pairs in $(\boldsymbol{\alpha}, \boldsymbol{\beta})$ that are both finite and $r_{2}=r-r_{1}$. Similarly, $\boldsymbol{\kappa}=\left(\boldsymbol{\kappa}_{1}^{\top}, \boldsymbol{\kappa}_{2}^{\top}\right)^{\top}$ is partitioned as well. If $r_{1}=r$, then the truncation limits $\boldsymbol{\alpha}$ and $\boldsymbol{\beta}$ contains only finite elements, and hence $\mathbb{E}\left[\mathbf{X}^{\kappa} \mid \boldsymbol{\alpha} \leq \mathbf{X} \leq \boldsymbol{\beta}\right]$ exists for all $\boldsymbol{\kappa} \in \mathbb{N}^{r}$ because the distribution is bounded. When $r_{2} \geq 1$, there exists at least one pair in $(\boldsymbol{\alpha}, \boldsymbol{\beta})$ containing infinite values, and the expectation may not exist. Given that $\mathbb{E}\left[\mathbf{X}^{\kappa} \mid \boldsymbol{\alpha} \leq \mathbf{X} \leq \boldsymbol{\beta}\right]=\mathbb{E}\left[\mathbf{X}_{1}^{\boldsymbol{\kappa}_{1}} \mathbb{E}\left[\mathbf{X}_{2}^{\boldsymbol{\kappa}_{2}} \mid \mathbf{X}_{1}, \boldsymbol{\alpha}_{2} \leq \mathbf{X}_{2} \leq \boldsymbol{\beta}_{2}\right] \mid \boldsymbol{\alpha}_{1} \leq \mathbf{X}_{1} \leq \boldsymbol{\beta}_{1}\right]$, for any measurable function $g, \mathbb{E}\left[g\left(\mathbf{X}_{1}\right) \mid \boldsymbol{\alpha}_{1} \leq \mathbf{X}_{1} \leq \boldsymbol{\beta}_{1}\right]$ always exists, and $\left(\boldsymbol{\alpha}_{2}, \boldsymbol{\beta}_{2}\right)$ is not bounded, it is straightforward to see that $\mathbb{E}\left[\mathbf{X}^{\kappa} \mid \boldsymbol{\alpha} \leq \mathbf{X} \leq \boldsymbol{\beta}\right]$ exist if and only if (iff) the inner expectation $\mathbb{E}\left[\mathbf{X}_{2}^{\kappa_{2}} \mid \mathbf{X}_{1}\right]$ exists. 
As seen, the existence only depends of the order of the moment $\boldsymbol{\kappa}_{2}$ and the distribution of $\mathbf{X}_{2} \mid \mathbf{X}_{1}$, this last depending on the conditional generating function $h_{\mathbf{X}_{1}}^{\left(r_{2}\right)}$.

If $\mathbf{Y} \sim S L C T-E C_{p, q}\left(\boldsymbol{\xi}, \boldsymbol{\Omega}, h^{(q+p)}, C\right)$, with truncation subset of the form $C(\mathbf{c}, \mathbf{d})$ and $r=p+q$ say. It follows from Theorem 11 that $\mathbb{E}\left[\mathbf{Y}^{\mathbf{k}} \mid \mathbf{a} \leq \mathbf{Y} \leq \mathbf{b}\right]=\mathbb{E}\left[\mathbf{X}^{\kappa} \mid \boldsymbol{\alpha} \leq \mathbf{X} \leq \boldsymbol{\beta}\right]$. Hence, the same condition holds taking in account that $\boldsymbol{\kappa}=\left(\mathbf{0}_{q}^{\top}, \mathbf{k}^{\top}\right)^{\top}, \boldsymbol{\alpha}=\left(\mathbf{c}^{\top}, \mathbf{a}^{\top}\right)^{\top}$ and $\boldsymbol{\beta}=\left(\mathbf{d}^{\top}, \mathbf{b}^{\top}\right)^{\top}$. Next, we present a result for a particular case.

\section{The doubly truncated SUT distribution}

For the rest of the paper we shall focus our attention on the computation of the moments of the doubly truncated unified skew- $t$ (TSUT) distribution, denoted by $\mathbf{W} \sim \operatorname{TSUT}_{p, q}(\boldsymbol{\mu}, \boldsymbol{\Sigma}, \boldsymbol{\Lambda}, \boldsymbol{\tau}, \nu, \boldsymbol{\Sigma} ;(\mathbf{a}, \mathbf{b}))$. Besides, we shall study some of its properties and for its particular case (when $q=1$ ), the doubly truncated extended skew- $t$ distribution, say $\mathbf{W} \sim T E S T_{p}(\boldsymbol{\mu}, \boldsymbol{\Sigma}, \boldsymbol{\lambda}, \tau, \nu ;(\mathbf{a}, \mathbf{b}))$. For the limiting symmetrical case, we shall use the notation $\mathbf{W} \sim T t_{p}(\boldsymbol{\mu}, \boldsymbol{\Sigma}, \nu ;(\mathbf{a}, \mathbf{b}))$ to refer to a $p$-variate truncated Student- $t(\mathrm{TT})$ distribution on $(\mathbf{a}, \mathbf{b}) \in \mathbb{R}^{p}$. Finally, $\mathbf{W} \sim T N_{p}(\boldsymbol{\mu}, \boldsymbol{\Sigma} ;(\mathbf{a}, \mathbf{b}))$ will stand for a $p$-variate truncated normal distribution on the interval $(\mathbf{a}, \mathbf{b})$. Hereinafter we shall omit the expression doubly due to we only work with intervalar truncation.

Corollary 2 (moments of a TSUT). If $\mathbf{Y} \sim S U T_{p, q}(\boldsymbol{\mu}, \boldsymbol{\Sigma}, \boldsymbol{\Lambda}, \boldsymbol{\tau}, \nu, \boldsymbol{\Psi})$, it follows from Theorem $\square$ that

$$
\mathbb{E}\left[\mathbf{Y}^{\mathbf{k}} \mid \mathbf{a} \leq \mathbf{Y} \leq \mathbf{b}\right]=\mathbb{E}\left[\mathbf{X}^{\kappa} \mid \boldsymbol{\alpha} \leq \mathbf{X} \leq \boldsymbol{\beta}\right],
$$

where $\mathbf{X} \sim t_{q+p}(\boldsymbol{\xi}, \boldsymbol{\Omega}, \nu)$ with $\boldsymbol{\xi}$ and $\boldsymbol{\Omega}$ as defined in Equation (13) and $\boldsymbol{\kappa}=\left(\mathbf{0}_{q}^{\top}, \mathbf{k}^{\top}\right)^{\top}, \boldsymbol{\alpha}=\left(\mathbf{0}_{q}^{\top}, \mathbf{a}^{\top}\right)^{\top}$ and $\boldsymbol{\beta}=\left(\infty_{q}^{\top}, \mathbf{b}^{\top}\right)^{\top}$.

\subsection{Mean and covariance matrix of the TSUT distribution}

Let $\mathbf{Y} \sim T S U T_{p, q}(\boldsymbol{\mu}, \boldsymbol{\Sigma}, \boldsymbol{\Lambda}, \boldsymbol{\tau}, \nu, \boldsymbol{\Psi} ;(\mathbf{a}, \mathbf{b}))$ and $\mathbf{X} \sim T t_{q+p}(\boldsymbol{\xi}, \boldsymbol{\Omega}, \nu ;(\boldsymbol{\alpha}, \boldsymbol{\beta}))$. From Corollary 2 , we have that the first two moments of $\mathbf{Y}$ can be computed as

$$
\begin{aligned}
\mathbb{E}[\mathbf{Y}] & =\mathbf{m}_{2}, \\
\mathbb{E}\left[\mathbf{Y} \mathbf{Y}^{\top}\right] & =\mathbf{M}_{22},
\end{aligned}
$$

where $\mathbf{m}=\mathbb{E}[\mathbf{X}]$ and $\mathbf{M}=\mathbb{E}\left[\mathbf{X X} \mathbf{X}^{\top}\right]$ are partitioned as in Corollary 1 Notice that $\operatorname{cov}[\mathbf{Y}]=\mathbb{E}\left[\mathbf{Y} \mathbf{Y}^{\top}\right]-\mathbb{E}[\mathbf{Y}] \mathbb{E}\left[\mathbf{Y}^{\top}\right]$. Equations (27) and (28) are convenient for computing $\mathbb{E}[\mathbf{Y}]$ and $\operatorname{cov}[\mathbf{Y}]$ since all boils down to compute the mean and the variance-covariance matrix for a $q+p$-variate TT distribution which can be calculated using the our MomTrunc $\mathrm{R}$ package available on CRAN.

\section{Existence of the moments of a TSUT}

Let also $p_{1}$ be the number of pairs in $(\mathbf{a}, \mathbf{b})$ that are both finite. Without loss of generality, we assume $\mathbf{Y}=$ $\left(\mathbf{Y}_{1}^{\top}, \mathbf{Y}_{2}^{\top}\right)^{\top}$, where the upper and lower truncation limits associated with $\mathbf{Y}_{1}$ are both finite, but at least one of the truncation limits associated with $\mathbf{Y}_{2}$ is not finite, say $\operatorname{dim}\left(\mathbf{Y}_{1}\right)=p_{1}$ and $\operatorname{dim}\left(\mathbf{Y}_{2}\right)=p_{2}$, with $p_{1}+p_{2}=p$. Consider the partitions of $\mathbf{a}=\left(\mathbf{a}_{1}^{\top}, \mathbf{a}_{2}^{\top}\right)^{\top}, \mathbf{b}=\left(\mathbf{b}_{1}^{\top}, \mathbf{b}_{2}^{\top}\right)^{\top}$ and $\mathbf{k}=\left(\mathbf{k}_{1}^{\top}, \mathbf{k}_{2}^{\top}\right)^{\top}$ as well. The next proposition gives a sufficient condition for the existence of the moment of a TSUT distribution.

Proposition 2 (existence of the moments of a TSUT). Under the conditions above, $\mathbb{E}\left[\mathbf{Y}^{\mathrm{k}} \mid \mathbf{a} \leq \mathbf{Y} \leq \mathbf{b}\right]$ exists iff $\operatorname{sum}\left(\mathbf{k}_{2}\right)<\nu+p_{1}$.

Proof. From subsection 3.4 it is suffices to demonstrate that $\mathbb{E}\left[\mathbf{X}_{2}^{\kappa_{2}} \mid \mathbf{X}_{1}\right]$ exists. Since $\boldsymbol{\alpha}=\left(\mathbf{0}_{q}^{\top}, \mathbf{a}_{1}^{\top}, \mathbf{a}_{2}^{\top}\right)^{\top}$ and $\boldsymbol{\beta}=\left(\boldsymbol{\infty}_{q}^{\top}, \mathbf{b}_{1}^{\top}, \mathbf{b}_{2}^{\top}\right)^{\top}$, it follows that $r_{1}=p_{1}, r_{2}=q+p_{2}, \boldsymbol{\kappa}_{1}=\mathbf{k}_{1}$ and $\boldsymbol{\kappa}_{2}=\left(\mathbf{0}_{q}^{\top}, \mathbf{k}_{2}^{\top}\right)^{\top}$. It is easy to show that the distribution of $\mathbf{X}_{2} \mid \mathbf{X}_{1}$ is a $\left(q+p_{2}\right)$-variate Student- $t$ distribution with $\nu+p_{1}$ degrees of freedom. Hence, the above expectation exists iff $\operatorname{sum}\left(\mathbf{k}_{2}\right)<\nu+p_{1}$.

From Proposition 2, see that $\mathbb{E}[\mathbf{Y}]$ and $\mathbb{E}\left[\mathbf{Y Y}^{\top}\right]$ exist iff $\nu+p_{1}>1$ and $\nu+p_{1}>2$ respectively.

Remark 2 (Sufficient condition of existence of the first two moments of a TSUT). Since $\nu>0$, it is equivalent to say that, the first moment exists if at least one dimension containing a finite limit exists. Besides, the second moment exists if at least two dimensions containing a finite limit exist. 

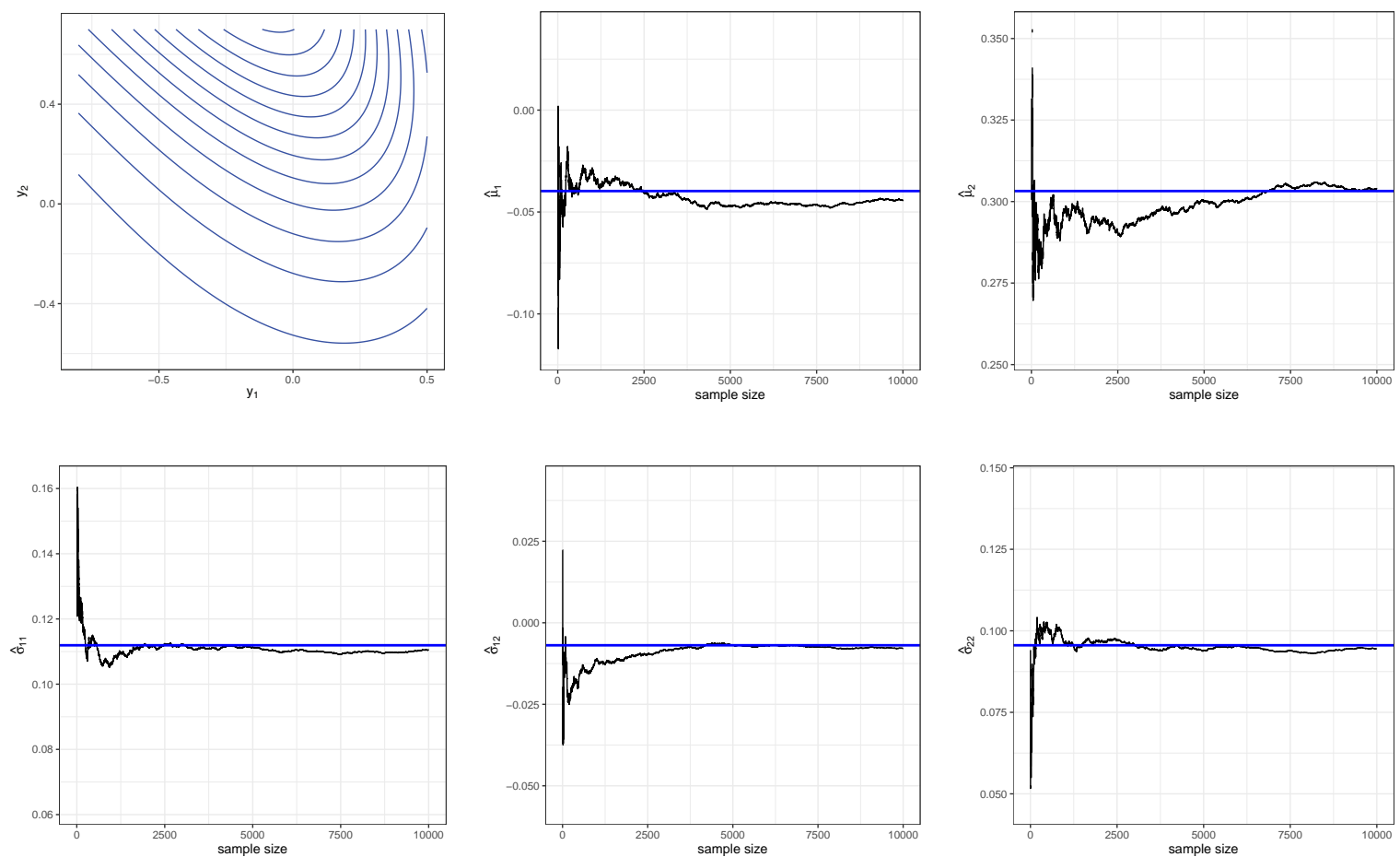

Figure 2: Simulation study. Contour plot for the TSUT density (upper left corner) and trace plots of the evolution of the MC estimates for the mean and variance-covariance elements of $\mathbf{Y}$. The solid line represent the true estimated value by our proposal.

Remark 3. Sufficient conditions aforementioned hold for the truncated Student- $t(q=0)$ and for the truncated EST distribution $(q=1)$ due to the condition does not depend on $q$.

Next, in light of proposition 1, we propose a corollary for the limiting case of a SUT pdf when $\tau \rightarrow-\infty$.

Corollary 3. Under the condition of Proposition 1, as $\tau \rightarrow-\infty$, i.e., $\tau_{i} \rightarrow-\infty, i=1, \ldots, q$, then

$$
S U T_{p, q}(\boldsymbol{\mu}, \boldsymbol{\Sigma}, \boldsymbol{\Lambda}, \boldsymbol{\tau}, \nu, \boldsymbol{\Psi}) \longrightarrow t_{p}\left(\boldsymbol{\gamma}, \omega_{\tau} \boldsymbol{\Gamma}, \nu+q\right),
$$

with $\boldsymbol{\gamma}=\boldsymbol{\mu}-\boldsymbol{\Omega}_{21} \boldsymbol{\Omega}_{11}^{-1} \boldsymbol{\tau}, \boldsymbol{\Gamma}=\boldsymbol{\Sigma}-\boldsymbol{\Omega}_{21} \boldsymbol{\Omega}_{11}^{-1} \boldsymbol{\Omega}_{12}$ and $\omega_{\tau}=\nu_{\mathbf{x}_{1}}^{2}(\mathbf{0})=\left(\nu+\boldsymbol{\tau}^{\top} \boldsymbol{\Omega}_{11}^{-1} \boldsymbol{\tau}\right) /(\nu+q)$ with $\boldsymbol{\Omega}_{11}=\mathbf{\Psi}+\boldsymbol{\Lambda}^{\top} \boldsymbol{\Lambda}$. In particular, for $q=1$,

$$
\operatorname{EST}_{p}(\boldsymbol{\mu}, \boldsymbol{\Sigma}, \boldsymbol{\lambda}, \tau, \nu) \longrightarrow t_{p}\left(\boldsymbol{\gamma},\left(\nu+\tilde{\tau}^{2}\right) /(\nu+1) \boldsymbol{\Gamma}, \nu+1\right),
$$

with $\gamma=\boldsymbol{\mu}-\tilde{\tau} \boldsymbol{\Delta}, \boldsymbol{\Gamma}=\boldsymbol{\Sigma}-\boldsymbol{\Delta} \boldsymbol{\Delta}^{\top}$, and $\boldsymbol{\Delta}=\boldsymbol{\Sigma}^{1 / 2} \boldsymbol{\lambda} / \sqrt{1+\boldsymbol{\lambda}^{\top} \boldsymbol{\lambda}}$.

It is worth to stress that parameters $\Delta$ and $\boldsymbol{\Gamma}$ are well know in the context of SN and ST modeling since they are used in the the stochastic representation of this variates. Furthermore, the resulting symmetric distribution is highly involved in the framework of censored modeling as shown next in Section 6.

\section{Numerical example}

In order to illustrate our method, we performed a simple Monte Carlo (MC) simulation study to show how MC estimators for the mean vector and variance-covariance matrix elements converge to the real values computed by our method.

We consider a bivariate TSUT distribution $\mathbf{Y} \sim \operatorname{TSUT}_{2,2}(\boldsymbol{\mu}, \boldsymbol{\Sigma}, \boldsymbol{\Lambda}, \boldsymbol{\tau}, \nu, \boldsymbol{\Psi} ;(\mathbf{a}, \mathbf{b}))$ with lower and upper truncation limits $\mathbf{a}=(-0.8,-0.6)^{\top}$ and $\mathbf{b}=(0.5,0.7)^{\top}$ respectively, null location vector $\boldsymbol{\mu}=\mathbf{0}$, degrees of freedom $\nu=4$,

$$
\boldsymbol{\tau}=\left(\begin{array}{c}
-1 \\
2
\end{array}\right), \quad \boldsymbol{\Sigma}=\left(\begin{array}{cc}
1 & 0.2 \\
0.2 & 4
\end{array}\right), \quad \boldsymbol{\Lambda}=\left(\begin{array}{cc}
1 & 3 \\
-3 & -2
\end{array}\right) \quad \text { and } \quad \boldsymbol{\Psi}=\left(\begin{array}{cc}
1 & -0.5 \\
-0.5 & 1
\end{array}\right) .
$$


Figure 2 shows the contour plot for the TSUT density (upper left corner) as well as the evolution trace of the MC estimates for the mean (first row) and variance-covariance (last row) elements $\mu_{1}, \mu_{2}, \sigma_{11}, \sigma_{12}$ and $\sigma_{22}$. Estimated true values for the mean vector and the variance-covariance matrix were computed using equations (27) and (28), being

$$
\mathbb{E}[\mathbf{Y}]=\left(\begin{array}{c}
-0.039 \\
0.303
\end{array}\right) \quad \text { and } \quad \operatorname{cov}[\mathbf{Y}]=\left(\begin{array}{cc}
0.112 & -0.007 \\
-0.007 & 0.096
\end{array}\right)
$$

which are depicted as a blue solid line in Figure 2. Note that even with $1000 \mathrm{MC}$ simulations there exists a significant variation in the chains.

\section{Additional results related to interval censored mechanism}

Under interval censoring mechanism the implementation of inferences depends on the computation of certain marginal and conditional expectations ([17]). For instance, for $\mathbf{X}=\left(\mathbf{X}_{1}^{\top}, \mathbf{X}_{2}^{\top}\right)^{\top} \sim \phi_{1+p}(\boldsymbol{\xi}, \boldsymbol{\Omega}, \nu)$, as in [13), with $\boldsymbol{\Psi}=1$, $\boldsymbol{\Lambda}=\boldsymbol{\lambda}$ and $\boldsymbol{\tau}=0$, it holds that $f_{\mathbf{X}_{1}}\left(\mathbf{0} \mid \mathbf{X}_{2}=\mathbf{Y}\right)=\phi\left(\boldsymbol{\lambda}^{\top} \boldsymbol{\Sigma}^{-1 / 2}(\mathbf{Y}-\boldsymbol{\mu})\right)$. Then,

$$
\mathbb{E}\left[g(\mathbf{Y}) \frac{f_{\mathbf{X}_{1}}\left(\mathbf{0} \mid \mathbf{X}_{2}=\mathbf{Y}\right)}{\mathbb{P}\left(\mathbf{X}_{1}>\mathbf{0} \mid \mathbf{X}_{2}=\mathbf{Y}\right)}\right]=\mathbb{E}\left[g(\mathbf{Y}) \frac{\phi\left(\boldsymbol{\lambda}^{\top} \boldsymbol{\Sigma}^{-1 / 2}(\mathbf{Y}-\boldsymbol{\mu})\right)}{\Phi\left(\boldsymbol{\lambda}^{\top} \boldsymbol{\Sigma}^{-1 / 2}(\mathbf{Y}-\boldsymbol{\mu})\right)}\right],
$$

where $g(\cdot)$ is a measurable function. The expectation in the right side of the expression (31) is highly used to perform inferences under SN censored models from a likelihood-based perspective, such as the E-Step of the EM-algorithm ([18]).

Next, we derive general expressions that are involved in interval censored modeling, specifically, in the E-step of the EM algorithm. These expressions arise, when we consider the responses $\mathbf{Y}_{i}, i=1, \ldots, n$, to be i.i.d. realizations from a selection elliptical distribution or any of its particular cases. For instance, a SUT, EST or ST distribution or any normal limiting case as the SUN, ESN or SN distribution as the example in 31.

Lemma 1. Let $\mathbf{X}=\left(\mathbf{X}_{1}^{\top}, \mathbf{X}_{2}^{\top}\right)^{\top} \sim E C_{q+p}\left(\boldsymbol{\xi}, \boldsymbol{\Omega}, h^{(q+p)}\right)$ and $\mathbf{Y} \sim T S L C T-E C_{p, q}\left(\boldsymbol{\xi}, \boldsymbol{\Omega}, h^{(q+p)}, C ;(\mathbf{a}, \mathbf{b})\right)$ with truncation subset $C=C(\mathbf{0})$. For any measurable function $g(\mathbf{y}): \mathbb{R}^{p} \rightarrow \mathbb{R}$, we have that

$$
\mathbb{E}\left[g(\mathbf{Y}) \frac{f_{\mathbf{X}_{1}}\left(\mathbf{0} \mid \mathbf{X}_{2}=\mathbf{Y}\right)}{\mathbb{P}\left(\mathbf{X}_{1}>\mathbf{0} \mid \mathbf{X}_{2}=\mathbf{Y}\right)}\right]=\frac{\mathbb{P}\left(\mathbf{a} \leq \mathbf{W}_{0} \leq \mathbf{b}\right)}{\mathbb{P}\left(\mathbf{a} \leq \mathbf{Y}_{0} \leq \mathbf{b}\right)} \frac{\mathbb{E}[g(\mathbf{W})]}{\mathbb{P}\left(\mathbf{X}_{1} \geq \mathbf{0}\right)} f_{\mathbf{X}_{1}}(\mathbf{0}),
$$

where $\mathbf{X}_{1} \sim E C_{p}\left(\boldsymbol{\xi}_{1}, \boldsymbol{\Omega}_{11}, h^{(q)}\right), \mathbf{Y}_{0} \sim S L C T-E C_{p, q}\left(\boldsymbol{\xi}, \boldsymbol{\Omega}, h^{(q+p)}, C(\mathbf{0})\right), \mathbf{W}_{0} \sim E C_{p}\left(\boldsymbol{\xi}_{2}-\boldsymbol{\Omega}_{21} \boldsymbol{\Omega}_{11}^{-1} \boldsymbol{\xi}_{1}, \boldsymbol{\Omega}_{22}-\right.$ $\left.\boldsymbol{\Omega}_{21} \boldsymbol{\Omega}_{11}^{-1} \boldsymbol{\Omega}_{21}, h_{\mathbf{0}}^{(p)}\right)$ and $\mathbf{W} \stackrel{d}{=} \mathbf{W}_{0} \mid\left(\mathbf{a} \leq \mathbf{W}_{0} \leq \mathbf{b}\right)$.

Proof. Using basic probability theory, we have

$$
\begin{aligned}
& =\mathbb{E}\left[g(\mathbf{Y}) \frac{f_{\mathbf{X}_{1}}\left(\mathbf{0} \mid \mathbf{X}_{2}=\mathbf{Y}\right)}{\mathbb{P}\left(\mathbf{X}_{1}>\mathbf{0} \mid \mathbf{X}_{2}=\mathbf{Y}\right)}\right] \\
& =\frac{1}{\mathbb{P}\left(\mathbf{a} \leq \mathbf{Y}_{0} \leq \mathbf{b}\right)} \int_{\mathbf{a}}^{\mathbf{b}} g(\mathbf{y}) \frac{f_{\mathbf{X}_{1}}\left(\mathbf{0} \mid \mathbf{X}_{2}=\mathbf{y}\right)}{\mathbb{P}\left(\mathbf{X}_{1}>\mathbf{0} \mid \mathbf{X}_{2}=\mathbf{y}\right)} f_{\mathbf{Y}}(\mathbf{y}) \mathrm{d} \mathbf{y} \\
& =\frac{1}{\mathbb{P}\left(\mathbf{a} \leq \mathbf{Y}_{0} \leq \mathbf{b}\right)} \int_{\mathbf{a}}^{\mathbf{b}} g(\mathbf{y}) \frac{f_{\mathbf{X}_{1}}\left(\mathbf{0} \mid \mathbf{X}_{2}=\mathbf{y}\right)}{\mathbb{P}\left(\mathbf{X}_{1}>\mathbf{0} \mid \mathbf{X}_{2}=\mathbf{y}\right)} \frac{\mathbb{P}\left(\mathbf{X}_{1}>\mathbf{0} \mid \mathbf{X}_{2}=\mathbf{y}\right) f_{\mathbf{X}_{2}}(\mathbf{y})}{\mathbb{P}\left(\mathbf{X}_{1}>\mathbf{0}\right)} \mathrm{d} \mathbf{y} \\
& =\frac{1}{\mathbb{P}\left(\mathbf{a} \leq \mathbf{Y}_{0} \leq \mathbf{b}\right)} \int_{\mathbf{a}}^{\mathbf{b}} g(\mathbf{y}) \frac{f_{\mathbf{X}_{1}}\left(\mathbf{0} \mid \mathbf{X}_{2}=\mathbf{y}\right) f_{\mathbf{X}_{2}}(\mathbf{y})}{\mathbb{P}\left(\mathbf{X}_{1}>\mathbf{0}\right)} \mathrm{d} \mathbf{y} \\
& =\frac{1}{\mathbb{P}\left(\mathbf{a} \leq \mathbf{Y}_{0} \leq \mathbf{b}\right)} \frac{f_{\mathbf{X}_{1}(\mathbf{0})}}{\mathbb{P}\left(\mathbf{X}_{1}>\mathbf{0}\right)} \int_{\mathbf{a}}^{\mathbf{b}} g(\mathbf{y}) f_{\mathbf{X}_{2}}\left(\mathbf{y} \mid \mathbf{X}_{1}=\mathbf{0}\right) \mathrm{d} \mathbf{y} \\
& =\frac{\mathbb{P}\left(\mathbf{a} \leq \mathbf{W}_{0} \leq \mathbf{b}\right)}{\mathbb{P}\left(\mathbf{a} \leq \mathbf{Y}_{0} \leq \mathbf{b}\right)} \frac{\mathbb{E}[g(\mathbf{W})]}{\mathbb{P}\left(\mathbf{X}_{1}>\mathbf{0}\right)} f_{\mathbf{X}_{1}}(\mathbf{0})
\end{aligned}
$$

where $\mathbf{W}_{0} \stackrel{d}{=} \mathbf{X}_{2} \mid\left(\mathbf{X}_{1}=\mathbf{0}\right)$ and $\mathbf{W} \stackrel{d}{=} \mathbf{W}_{0} \mid\left(\mathbf{a} \leq \mathbf{W}_{0} \leq \mathbf{b}\right)$. 
Lemma 2. Consider $\mathbf{X}, \mathbf{Y}$ and $g$ as in Lemma $\square$ Now, consider $\mathbf{Y}$ to be partitioned as $\mathbf{Y}=\left(\mathbf{Y}_{1}^{\top}, \mathbf{Y}_{2}^{\top}\right)^{\top}$ of dimensions $p_{1}$ and $p_{2}\left(p_{1}+p_{2}=p\right)$. For a given random variable $\mathbf{U}$, let $\mathbf{U}^{*}$ stands for $\mathbf{U}^{*} \stackrel{d}{=} \mathbf{U} \mid \mathbf{Y}_{1}$. It follows that

$$
\mathbb{E}\left[g\left(\mathbf{Y}_{2}\right) \frac{f_{\mathbf{X}_{1}}\left(\mathbf{0} \mid \mathbf{X}_{2}=\mathbf{Y}\right)}{\mathbb{P}\left(\mathbf{X}_{1}>\mathbf{0} \mid \mathbf{X}_{2}=\mathbf{Y}\right)} \mid \mathbf{Y}_{1}\right]=\frac{\mathbb{P}\left(\mathbf{a}_{2} \leq \mathbf{W}_{0}^{*} \leq \mathbf{b}_{2}\right)}{\mathbb{P}\left(\mathbf{a}_{2} \leq \mathbf{Y}_{0}^{*} \leq \mathbf{b}_{2}\right)} \frac{\mathbb{E}\left[g\left(\mathbf{W}_{2}\right)\right]}{\mathbb{P}\left(\mathbf{X}_{1}^{*}>\mathbf{0}\right)} f_{\mathbf{X}_{1}^{*}}(\mathbf{0})
$$

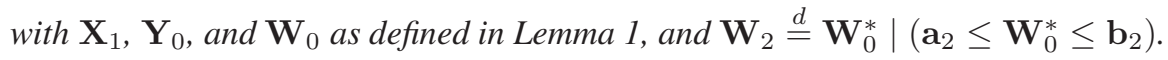

Proof. Consider $\mathbf{X}_{2}$ partitioned as $\mathbf{X}_{2}=\left(\mathbf{X}_{21}^{\top}, \mathbf{X}_{22}^{\top}\right)^{\top}$ such that $\operatorname{dim}\left(\mathbf{X}_{21}\right)=\operatorname{dim}\left(\mathbf{Y}_{1}\right)$ and $\operatorname{dim}\left(\mathbf{X}_{22}\right)=\operatorname{dim}\left(\mathbf{Y}_{2}\right)$. Since $f_{\mathbf{Y}_{2}}\left(\mathbf{y}_{2} \mid \mathbf{Y}_{1}=\mathbf{y}_{1}\right)=f_{\mathbf{Y}}(\mathbf{y}) / f_{\mathbf{Y}_{1}}\left(\mathbf{y}_{1}\right)$, it follows (in a similar manner that the proof of Lemma10 that

$$
\begin{aligned}
& =\mathbb{E}\left[g\left(\mathbf{Y}_{2}\right) \frac{f_{\mathbf{X}_{1}}\left(\mathbf{0} \mid \mathbf{X}_{2}=\mathbf{Y}\right)}{\mathbb{P}\left(\mathbf{X}_{1}>\mathbf{0} \mid \mathbf{X}_{2}=\mathbf{Y}\right)} \mid \mathbf{Y}_{1}\right] \\
& =\frac{1}{\mathbb{P}\left(\mathbf{a}_{2} \leq \mathbf{Y}_{0}^{*} \leq \mathbf{b}_{2}\right)} \int_{\mathbf{a}_{2}}^{\mathbf{b}_{2}} g\left(\mathbf{y}_{2}\right) \frac{f_{\mathbf{X}_{1}}\left(\mathbf{0} \mid \mathbf{X}_{2}=\mathbf{y}\right)}{\mathbb{P}\left(\mathbf{X}_{1}>\mathbf{0} \mid \mathbf{X}_{2}=\mathbf{y}\right)} \frac{\mathbb{P}\left(\mathbf{X}_{1}>\mathbf{0} \mid \mathbf{X}_{2}=\mathbf{y}\right)}{\mathbb{P}\left(\mathbf{X}_{1}>\mathbf{0} \mid \mathbf{X}_{21}=\mathbf{y}_{1}\right)} \frac{f_{\mathbf{X}_{2}}(\mathbf{y})}{f_{\mathbf{X}_{21}}\left(\mathbf{y}_{1}\right)} \mathrm{d} \mathbf{y}_{2}, \\
& =\frac{1}{\mathbb{P}\left(\mathbf{a}_{2} \leq \mathbf{Y}_{0}^{*} \leq \mathbf{b}_{2}\right)} \int_{\mathbf{a}_{2}}^{\mathbf{b}_{2}} g\left(\mathbf{y}_{2}\right) \frac{f_{\mathbf{X}_{1}}\left(\mathbf{0} \mid \mathbf{X}_{2}=\mathbf{y}\right)}{\mathbb{P}\left(\mathbf{X}_{1}>\mathbf{0} \mid \mathbf{X}_{21}=\mathbf{y}_{1}\right)} \frac{f_{\mathbf{X}_{2}}(\mathbf{y})}{f_{\mathbf{X}_{21}}\left(\mathbf{y}_{1}\right)} \mathrm{d}_{\mathbf{y}_{2}}, \\
& =\frac{1}{\mathbb{P}\left(\mathbf{a}_{2} \leq \mathbf{Y}_{0}^{*} \leq \mathbf{b}_{2}\right)} \frac{f_{\mathbf{X}_{1}}(\mathbf{0})}{\mathbb{P}\left(\mathbf{X}_{1}>\mathbf{0} \mid \mathbf{X}_{21}=\mathbf{y}_{1}\right)} \int_{\mathbf{a}_{2}}^{\mathbf{b}_{2}} g\left(\mathbf{y}_{2}\right) \frac{f_{\mathbf{X}_{2}}\left(\mathbf{y} \mid \mathbf{X}_{1}=\mathbf{0}\right)}{f_{\mathbf{X}_{21}}\left(\mathbf{y}_{1}\right)} \mathrm{d}_{\mathbf{y}_{2}}, \\
& =\frac{1}{\mathbb{P}\left(\mathbf{a}_{2} \leq \mathbf{Y}_{0}^{*} \leq \mathbf{b}_{2}\right)} \frac{f_{\mathbf{X}_{1}}\left(\mathbf{0} \mid \mathbf{X}_{21}=\mathbf{y}_{1}\right)}{\mathbb{P}\left(\mathbf{X}_{1}>\mathbf{0} \mid \mathbf{X}_{21}=\mathbf{y}_{1}\right)} \int_{\mathbf{a}_{2}}^{\mathbf{b}_{2}} g\left(\mathbf{y}_{2}\right) f_{\mathbf{X}_{22}}\left(\mathbf{y}_{2} \mid \mathbf{X}_{21}=\mathbf{y}_{1}, \mathbf{X}_{1}=\mathbf{0}\right) \mathrm{d} \mathbf{y}_{2}, \\
& =\frac{\mathbb{P}\left(\mathbf{a}_{2} \leq \mathbf{W}_{0}^{*} \leq \mathbf{b}_{2}\right)}{\mathbb{P}\left(\mathbf{a}_{2} \leq \mathbf{Y}_{0}^{*} \leq \mathbf{b}_{2}\right)} \frac{\mathbb{E}\left[g\left(\mathbf{W}_{2}\right)\right]}{\mathbb{P}\left(\mathbf{X}_{1}^{*}>\mathbf{0}\right)} f_{\mathbf{X}_{1}^{*}}(\mathbf{0}),
\end{aligned}
$$

where $\mathbf{W}_{0}^{*} \stackrel{d}{=} \mathbf{X}_{22} \mid\left(\mathbf{X}_{21}=\mathbf{y}_{1}, \mathbf{X}_{1}=\mathbf{0}\right)$ and $\mathbf{W}_{2} \stackrel{d}{=} \mathbf{W}_{0}^{*} \mid\left(\mathbf{a}_{2} \leq \mathbf{W}_{0}^{*} \leq \mathbf{b}_{2}\right)$.

In the next corollaries, we particularize the aforementioned lemmas to the truncated SUT, EST, SUN and ESN distributions.

Corollary 4. Under the condition of Lemma \ let $\mathbf{Y} \sim \operatorname{TSUT}_{p, q}(\boldsymbol{\mu}, \boldsymbol{\Sigma}, \boldsymbol{\Lambda}, \boldsymbol{\tau}, \nu, \boldsymbol{\Psi},(\mathbf{a}, \mathbf{b}))$. For any measurable function $g(\mathbf{y}): \mathbb{R}^{p} \rightarrow \mathbb{R}$, we have that

$$
\mathbb{E}\left[g(\mathbf{Y}) \frac{t_{q}\left(\left(\boldsymbol{\tau}+\boldsymbol{\Lambda}^{\top} \boldsymbol{\Sigma}^{-1 / 2}(\mathbf{Y}-\boldsymbol{\mu})\right) \nu(\mathbf{Y}), \boldsymbol{\Psi} ; \nu+p\right)}{T_{q}\left(\left(\boldsymbol{\tau}+\boldsymbol{\Lambda}^{\top} \boldsymbol{\Sigma}^{-1 / 2}(\mathbf{Y}-\boldsymbol{\mu})\right) \nu(\mathbf{Y}), \mathbf{\Psi} ; \nu+p\right)}\right]=\frac{\mathbb{P}\left(\mathbf{a} \leq \mathbf{W}_{0} \leq \mathbf{b}\right)}{\mathbb{P}\left(\mathbf{a} \leq \mathbf{Y}_{0} \leq \mathbf{b}\right)} \mathbb{E}[g(\mathbf{W})] \boldsymbol{\eta},
$$

where $\boldsymbol{\eta}=t_{q}\left(\boldsymbol{\tau} ; \boldsymbol{\Psi}+\boldsymbol{\Lambda}^{\top} \boldsymbol{\Lambda}, \nu\right) / T_{q}\left(\boldsymbol{\tau} ; \boldsymbol{\Psi}+\boldsymbol{\Lambda}^{\top} \boldsymbol{\Lambda}, \nu\right), \mathbf{Y}_{0} \sim S U T_{p, q}(\boldsymbol{\mu}, \boldsymbol{\Sigma}, \boldsymbol{\Lambda}, \boldsymbol{\tau}, \nu, \boldsymbol{\Psi}), \mathbf{W}_{0} \sim t_{p}\left(\boldsymbol{\gamma}, \omega_{\tau} \boldsymbol{\Gamma}, \nu+q\right)$ and $\mathbf{W} \stackrel{d}{=} \mathbf{W}_{0} \mid\left(\mathbf{a} \leq \mathbf{W}_{0} \leq \mathbf{b}\right)$. When $\boldsymbol{\tau}=\mathbf{0}$, we have that $\boldsymbol{\eta}=2 t_{q}\left(\boldsymbol{\tau} ; \boldsymbol{\Psi}+\boldsymbol{\Lambda}^{\top} \boldsymbol{\Lambda}, \nu\right)$ and $\mathbf{W}_{0} \sim t_{p}(\boldsymbol{\mu}, \nu \boldsymbol{\Gamma} /(\nu+q), \nu+q)$.

In particular for $q=1, \mathbf{Y} \sim \operatorname{TEST}_{p}(\boldsymbol{\mu}, \boldsymbol{\Sigma}, \boldsymbol{\lambda}, \tau, \nu ;(\mathbf{a}, \mathbf{b}))$, and

$$
\mathbb{E}\left[g(\mathbf{Y}) \frac{t_{1}\left(\left(\tau+\boldsymbol{\lambda}^{\top} \boldsymbol{\Sigma}^{-1 / 2}(\mathbf{Y}-\boldsymbol{\mu})\right) \nu(\mathbf{Y}) ; \nu+p\right)}{T_{1}\left(\left(\tau+\boldsymbol{\lambda}^{\top} \boldsymbol{\Sigma}^{-1 / 2}(\mathbf{Y}-\boldsymbol{\mu})\right) \nu(\mathbf{Y}) ; \nu+p\right)}\right]=\frac{\mathbb{P}\left(\mathbf{a} \leq \mathbf{W}_{0} \leq \mathbf{b}\right)}{\mathbb{P}\left(\mathbf{a} \leq \mathbf{Y}_{0} \leq \mathbf{b}\right)} \eta \mathbb{E}[g(\mathbf{W})],
$$

with $\eta=t_{1}\left(\tau ; 1+\boldsymbol{\lambda}^{\top} \boldsymbol{\lambda}, \nu\right) / T_{1}(\tilde{\tau} ; \nu), \mathbf{Y}_{0} \sim \operatorname{EST}_{p}(\boldsymbol{\mu}, \boldsymbol{\Sigma}, \boldsymbol{\lambda}, \boldsymbol{\tau}, \nu), \mathbf{W}_{0} \sim t_{p}\left(\boldsymbol{\gamma},\left(\nu+\tilde{\tau}^{2}\right) \boldsymbol{\Gamma} /(\nu+1), \nu+1\right)$, and $\mathbf{W} \stackrel{d}{=} \mathbf{W}_{0} \mid\left(\mathbf{a} \leq \mathbf{W}_{0} \leq \mathbf{b}\right)$. Similarly, when $\tau=0$, we have that $\eta=2 t_{1}\left(0 ; 1+\boldsymbol{\lambda}^{\top} \boldsymbol{\lambda}, \nu\right)$ and $\mathbf{W}_{0} \sim t_{p}(\boldsymbol{\mu}, \nu \boldsymbol{\Gamma} /(\nu+$ $1), \nu+1)$.

Corollary 5. Under the condition of Lemma 1 let $\nu \rightarrow \infty, \mathbf{Y} \sim T \operatorname{TSU} N_{p, q}(\boldsymbol{\mu}, \boldsymbol{\Sigma}, \boldsymbol{\Lambda}, \boldsymbol{\tau}, \boldsymbol{\Psi},(\mathbf{a}, \mathbf{b}))$, it follows that

$$
\mathbb{E}\left[g(\mathbf{Y}) \frac{\phi_{q}\left(\boldsymbol{\tau}+\boldsymbol{\Lambda}^{\top} \boldsymbol{\Sigma}^{-1 / 2}(\mathbf{Y}-\boldsymbol{\mu}), \boldsymbol{\Psi}\right)}{\Phi_{q}\left(\boldsymbol{\tau}+\boldsymbol{\Lambda}^{\top} \boldsymbol{\Sigma}^{-1 / 2}(\mathbf{Y}-\boldsymbol{\mu}), \boldsymbol{\Psi}\right)}\right]=\frac{\mathbb{P}\left(\mathbf{a} \leq \mathbf{W}_{0} \leq \mathbf{b}\right)}{\mathbb{P}\left(\mathbf{a} \leq \mathbf{Y}_{0} \leq \mathbf{b}\right)} \mathbb{E}[g(\mathbf{W})] \boldsymbol{\eta},
$$


where $\boldsymbol{\eta}=\phi_{q}\left(\boldsymbol{\tau} ; \boldsymbol{\Psi}+\boldsymbol{\Lambda}^{\top} \boldsymbol{\Lambda}\right) / \Phi_{q}\left(\boldsymbol{\tau} ; \boldsymbol{\Psi}+\boldsymbol{\Lambda}^{\top} \boldsymbol{\Lambda}\right), \mathbf{Y}_{0} \sim S U N_{p, q}(\boldsymbol{\mu}, \boldsymbol{\Sigma}, \boldsymbol{\Lambda}, \boldsymbol{\tau}, \boldsymbol{\Psi}), \mathbf{W}_{0} \sim N_{p}(\boldsymbol{\gamma}, \boldsymbol{\Gamma})$, and $\mathbf{W} \stackrel{d}{=} \mathbf{W}_{0} \mid\left(\mathbf{a} \leq \mathbf{W}_{0} \leq \mathbf{b}\right)$. When $\boldsymbol{\tau}=\mathbf{0}$, we have that $\boldsymbol{\eta}=2 \phi_{q}\left(\mathbf{0} ; \boldsymbol{\Psi}+\boldsymbol{\Lambda}^{\top} \boldsymbol{\Lambda}\right)$ and $\mathbf{W}_{0} \sim N_{p}(\boldsymbol{\mu}, \boldsymbol{\Gamma})$.

In particular for $q=1, \mathbf{Y} \sim \operatorname{TESN}_{p}(\boldsymbol{\mu}, \boldsymbol{\Sigma}, \boldsymbol{\lambda} ;(\mathbf{a}, \mathbf{b}))$, and

$$
\mathbb{E}\left[g(\mathbf{Y}) \frac{\phi\left(\tau+\boldsymbol{\lambda}^{\top} \boldsymbol{\Sigma}^{-1 / 2}(\mathbf{Y}-\boldsymbol{\mu})\right)}{\Phi\left(\tau+\boldsymbol{\lambda}^{\top} \boldsymbol{\Sigma}^{-1 / 2}(\mathbf{Y}-\boldsymbol{\mu})\right)}\right]=\frac{\mathbb{P}\left(\mathbf{a} \leq \mathbf{W}_{0} \leq \mathbf{b}\right)}{\mathbb{P}\left(\mathbf{a} \leq \mathbf{Y}_{0} \leq \mathbf{b}\right)} \eta \mathbb{E}[g(\mathbf{W})],
$$

with $\eta=\phi\left(\tau ; 1+\boldsymbol{\lambda}^{\top} \boldsymbol{\lambda}\right) / \Phi(\tilde{\tau}), \mathbf{Y}_{0} \sim \operatorname{ESN}_{p}(\boldsymbol{\mu}, \boldsymbol{\Sigma}, \boldsymbol{\lambda}, \boldsymbol{\tau}), \mathbf{W}_{0} \sim N_{p}(\boldsymbol{\gamma}, \boldsymbol{\Gamma})$, and $\mathbf{W} \stackrel{d}{=} \mathbf{W}_{0} \mid\left(\mathbf{a} \leq \mathbf{W}_{0} \leq \mathbf{b}\right)$. Similarly, when $\tau=0$, we have that $\eta=\sqrt{2 / \pi\left(1+\boldsymbol{\lambda}^{\top} \boldsymbol{\lambda}\right)}$ and $\mathbf{W}_{0} \sim N_{p}(\boldsymbol{\mu}, \boldsymbol{\Gamma})$.

\section{Application of SE truncated moments on tail conditional expectation}

Let $Y$ be a random variable representing in this context, the total loss in a portfolio investment, a credit score, etc. Let $y_{\alpha}$ be the $(1-\alpha)$ th quantile of $Y$, that is, $\mathbb{P}\left(Y>y_{\alpha}\right)=\alpha$. Hence, the tail conditional expectation (TCE) (see, e.g., [19]) is denoted by

$$
\operatorname{TCE}_{Y}\left(y_{\alpha}\right)=\mathbb{E}\left[Y \mid Y>y_{\alpha}\right] .
$$

This can be interpreted as the expected value of the $\alpha \%$ worse losses. The quantile $y_{\alpha}$ is usually chosen to be high in order to be pessimistic, for instance, $\alpha=0.05$. Notice that, if we consider a variable $Y$ which we are interested on maximizing, for example, the pay-off of a portfolio, we simply compute $T C E_{-Y}\left(-y_{\alpha}\right)=-\mathbb{E}\left[Y \mid Y \leq-y_{\alpha}\right]$, being a measure of worst expected income.

Main applications of TCE are in actuarial science and financial economics: market risk, credit risk of a portfolio, insurance, capital requirements for financial institutions, among others. TCE (also known as tail value at risk, TVaR) and it represents an alternative to the traditional value at risk $(\mathrm{VaR})$ that is more sensitive to the shape of the tail of the loss distribution. Furthermore, if $Y$ is a continuous r.v., TCE coincides with the well-known risk measure expected shortfall ([20]). In contrast with VaR, TCE is said to be a coherent measure, holding desirable mathematical properties in the context of risk measurement and and is a convex function of the selection weights ([21, 22]). A good reference to several risk measures and their properties can be found in [23].

Multivariate framework Let consider a set of $p$ assets, business lines, credit scores, $\mathbf{Y}=\left(Y_{1}, \cdots, Y_{p}\right)^{\top}$. In the multivariate case, the sum of risks arises as a natural and simple measure of total risk. Hence, the sum $S=$ $Y_{1}+Y_{2}+\cdots+Y_{p}$ follows a univariate distribution and from (38), we have that the TCE for $S$ is given by

$$
T C E_{S}\left(s_{\alpha}\right)=\mathbb{E}\left[S \mid S>s_{\alpha}\right] .
$$

Even though we may know the marginal distribution of $S$, it is preferable to compute the total risk $T C E$ of $S$ as a decomposed sum, that is

$$
\mathbb{E}\left[S \mid S>s_{\alpha}\right]=\sum_{i=1}^{p} \mathbb{E}\left[Y_{i} \mid S>s_{\alpha}\right],
$$

where each term $\mathbb{E}\left[Y_{i} \mid S>s_{\alpha}\right]$ represents the average amount of risk due to $Y_{i}$. This decomposed sum offers a way to study the individual impact of the elements of the set, being an improvement to (39).

In order to model combinations of correlated risks, [24] extended the TCE to the multivariate framework. The multivariate TCE (MTCE) is given by

$$
M T C E_{\mathbf{Y}}\left(\mathbf{y}_{\boldsymbol{\alpha}}\right)=\mathbb{E}\left[\mathbf{Y} \mid \mathbf{Y}>\mathbf{y}_{\boldsymbol{\alpha}}\right]=\mathbb{E}\left[\mathbf{Y} \mid Y_{1}>y_{1 \alpha_{1}}, \ldots, Y_{p}>y_{p \alpha_{p}}\right],
$$

with $\boldsymbol{\alpha}=\left(\alpha_{1}, \ldots, \alpha_{p}\right)$ be a vector of quantiles of interest. Notice that the quantile-level for the MTCE is fixed per each risk $i=1, \ldots, p$, in contrast with the TCE of the sum, which is fixed over all the sum of risk $S$.

\subsection{MTCE for selection elliptical distributions}

Let consider $\mathbf{Y} \sim S L C T-E C_{p, q}\left(\boldsymbol{\xi}, \boldsymbol{\Omega}, h^{(q+p)}, C\right)$. With loss of generality, we consider the selection subset $C=$ $C(\mathbf{0})$. It follows from Theorem 1 that

$$
\operatorname{MTCE} E_{\mathbf{Y}}\left(\mathbf{y}_{\boldsymbol{\alpha}}\right)=\mathbb{E}\left[\mathbf{X}_{2} \mid \mathbf{X}>\mathbf{x}_{\boldsymbol{\alpha}}\right]
$$


with $\mathbf{x}_{\boldsymbol{\alpha}}=\left(\mathbf{0}_{q}^{\top}, \mathbf{y}_{\boldsymbol{\alpha}}^{\top}\right)^{\top}$ and where $\mathbf{X}=\left(\mathbf{X}_{1}^{\top}, \mathbf{X}_{2}^{\top}\right)^{\top} \sim E C_{q+p}\left(\boldsymbol{\xi}, \boldsymbol{\Omega}, h^{(q+p)}\right)$. It is noteworthy that the computation of the MTCE for $\mathbf{Y}$ following a SE distribution relies on the calculation of truncated moments for its symmetrical elliptical multivariate case.

On the other hand, by noticing that $S=\mathbf{1}^{\top} \mathbf{Y}$, it follows from (7) that $S$ is an univariate SE distribution given by $S \sim S L C T-E C_{1, q}\left(\boldsymbol{\xi}_{s}, \boldsymbol{\Omega}_{s}, h^{(q+1)}, C\right)$, with

$$
\boldsymbol{\xi}_{S}=\left(\begin{array}{c}
\boldsymbol{\xi}_{1} \\
\mathbf{1}^{\top} \boldsymbol{\xi}_{2}
\end{array}\right) \quad \text { and } \quad \boldsymbol{\Omega}_{S}=\left(\begin{array}{cc}
\boldsymbol{\Omega}_{11} & \boldsymbol{\Omega}_{12} \mathbf{1} \\
\mathbf{1}^{\top} \boldsymbol{\Omega}_{21} & \mathbf{1}^{\top} \boldsymbol{\Omega}_{22} \mathbf{1}
\end{array}\right)
$$

Hence, its TCE in (39] can be easily computed as $\mathbb{E}\left[S \mid S>s_{\alpha}\right]=\mathbb{E}\left[\begin{array}{lllllll}W_{2} & \mathbf{W}_{1}>\mathbf{0}, W_{2}>s_{\alpha}\end{array}\right]$, $\mathbf{W}=\left(\mathbf{W}_{1}^{\top}, W_{2}\right)^{\top} \sim E C_{q+1}\left(\boldsymbol{\xi}_{s}, \boldsymbol{\Omega}_{s}, h^{(1+q)}\right)$, due to $S \stackrel{d}{=} W_{2} \mid\left(\mathbf{W}_{1}>\mathbf{0}\right)$. Next, we establish a general proposition for computing $\mathbb{E}\left[S \mid S>\alpha_{s}\right]$ in matrix form as a decomposed sum.

Proposition 3. Let $\mathbf{Y} \sim S L C T-E C_{p, q}\left(\boldsymbol{\xi}, \boldsymbol{\Omega}, h^{(q+p)}, C\right)$, with $\boldsymbol{\xi}$ and $\boldsymbol{\Omega}$ as in (44), and $\mathbf{W}=\left(\mathbf{W}_{1}^{\top}, W_{2}\right)^{\top} \sim$ $E C_{q+1}\left(\boldsymbol{\xi}_{S}, \boldsymbol{\Omega}_{S}, h^{(1+q)}\right)$ as before. It follows that

$$
\mathbb{E}\left[S \mid S>s_{\alpha}\right]=\mathbf{1}^{\top} \mathbf{s}
$$

with $\mathbf{s}=\boldsymbol{\xi}_{2}+\boldsymbol{\Omega}_{2 S} \boldsymbol{\Omega}_{S}^{-1}\left(\mathcal{E}_{S}-\boldsymbol{\xi}_{S}\right)$, where $\boldsymbol{\Omega}_{2 S}=\left(\boldsymbol{\Omega}_{21}, \boldsymbol{\Omega}_{22} \mathbf{1}\right)$ and $\mathcal{E}_{S}=\mathbb{E}\left[\mathbf{W} \mid \mathbf{W}_{1}>\mathbf{0}, W_{2}>s_{\alpha}\right]$.

Proof. Let $\mathbf{A}=\left(\mathbf{1}, \mathbf{I}_{p}\right)^{\top}$ be a real matrix of dimensions $(p+1) \times p$. For $\mathbf{V}=\mathbf{A Y}$, it follows that

$$
\mathbf{V}=\left(\begin{array}{c}
V_{1} \\
\mathbf{V}_{2}
\end{array}\right) \sim S L C T-E C_{p+1, q}\left(\boldsymbol{\xi}_{V}=\left(\begin{array}{c}
\boldsymbol{\xi}_{S} \\
\boldsymbol{\xi}_{2}
\end{array}\right), \boldsymbol{\Omega}_{V}=\left(\begin{array}{cc}
\boldsymbol{\Omega}_{S} & \boldsymbol{\Omega}_{2 S}^{\top} \\
\boldsymbol{\Omega}_{2 S} & \boldsymbol{\Omega}_{22}
\end{array}\right), h^{(q+1+p)}, C\right),
$$

where $\mathbf{V}=\left(S, \mathbf{Y}^{\top}\right)^{\top}$. It comes from the definition of selection distribution that $\mathbf{V} \stackrel{d}{=}\left(X_{2}, \mathbf{X}_{3}^{\top}\right)^{\top} \mid\left(\mathbf{X}_{1}>0\right)$, where $\mathbf{X}=\left(\mathbf{X}_{1}^{\top}, X_{2}, \mathbf{X}_{3}^{\top}\right)^{\top}$ is a partitioned random vector with elements of dimensions $q$, 1 and $p$ respectively, where $\mathbf{X} \sim E C_{p+q+1}\left(\boldsymbol{\xi}_{V}, \boldsymbol{\Omega}_{V} ; h^{(q+1+p)}\right)$. Hence, it is straightforward to see that

$$
\mathbf{s}=\mathbb{E}\left[\mathbf{Y} \mid S>s_{\alpha}\right]=\mathbb{E}\left[\mathbf{X}_{3} \mid \mathbf{X}_{1}>\mathbf{0}, X_{2}>s_{\alpha},-\infty \leq \mathbf{X}_{3} \leq \infty\right]
$$

Since there exists a non-truncated partition, the result in 43 then immediately follows from equation (23), with $\mathbf{W}=\left(\mathbf{X}_{1}, X_{2}\right)^{\top}$.

Remark 4. It is noteworthy that, the ith element of vector $\mathbf{s}$, say $s_{i}=\mathbf{e}_{i}^{\top} \mathbf{s}$, is equal to $\mathbb{E}\left[Y_{i} \mid S>\alpha_{s}\right]$, representing the contribution to the total risk due to the ith risk.

Remark 5. Since $S \stackrel{d}{=} W_{2} \mid\left(\mathbf{W}_{1}>\mathbf{0}\right)$, it follows that the last element of the vector $\mathcal{E}_{s}$ is equivalent to $\mathbb{E}[S \mid S>$ $\left.s_{\alpha}\right]=\mathbb{E}\left[W_{2} \mid \mathbf{W}_{1}>\mathbf{0}, W_{2}>s_{\alpha}\right]$.

\subsection{Application of MTCE using a ST distribution}

Suppose that a set of risks $\mathbf{Y}$ are distributed as $\mathbf{Y} \sim S T_{p}(\boldsymbol{\mu}, \boldsymbol{\Sigma}, \boldsymbol{\lambda}, \nu)$. Let $\mathbf{y}$ represents a realization of $\mathbf{Y}$. Based on $\mathbf{y}$, the set of parameters $\boldsymbol{\theta}=(\boldsymbol{\mu}, \boldsymbol{\Sigma}, \boldsymbol{\lambda}, \nu)^{\top}$ can be estimated through maximum likelihood estimation. It follows that

$$
\operatorname{MTCE} E_{\mathbf{Y}}\left(\mathbf{y}_{\boldsymbol{\alpha}}\right)=\mathbb{E}\left[\mathbf{X}_{2} \mid X_{1}>0, \mathbf{X}_{2}>\mathbf{y}_{\boldsymbol{\alpha}}\right],
$$

where $\mathbf{X}=\left(X_{1}, \mathbf{X}_{2}^{\top}\right)^{\top} \sim t_{1+p}(\boldsymbol{\xi}, \boldsymbol{\Omega}, \nu)$ with

$$
\xi=\left(\begin{array}{c}
0 \\
\mu
\end{array}\right) \quad \text { and } \quad \Omega=\left(\begin{array}{cc}
1 & \Delta^{\top} \\
\Delta & \Sigma
\end{array}\right)
$$

Additionally, using simple algebraic manipulation, it follows from (7) that

$$
S \sim S T_{1}\left(\mu_{S}=\sum_{i=1}^{p} \mu_{i}, \sigma_{S}^{2}=\sum_{i=1}^{p} \sum_{j=1}^{p} \sigma_{i j}, \lambda_{S}=\frac{\Delta_{S}}{\sqrt{\sigma_{S}^{2}-\Delta_{S}^{2}}}, \nu\right)
$$


with $\Delta_{S}=\sum_{i=1}^{p} \Delta_{i}$. Besides, the TCE of the sum is given by $T C E_{S}\left(s_{\alpha}\right)=\mathbb{E}\left[W_{2} \mid W_{1}>0, W_{2}>s_{\alpha}\right]$, $\mathbf{W}=\left(W_{1}^{\top}, W_{2}\right)^{\top} \sim t_{2}\left(\boldsymbol{\xi}_{S}, \boldsymbol{\Omega}_{S}, \nu\right)$, where

$$
\boldsymbol{\xi}_{S}=\left(\begin{array}{c}
0 \\
\mu_{S}
\end{array}\right), \quad \text { and } \quad \boldsymbol{\Omega}_{S}=\left(\begin{array}{cc}
1 & \Delta_{S} \\
\Delta_{S} & \sigma_{S}^{2}
\end{array}\right)
$$

Finally, we have from Proposition 3 that

$$
\begin{aligned}
\mathbb{E}\left[Y_{i} \mid S>\alpha_{s}\right], & =\mathbf{e}_{i}^{\top}\left[\boldsymbol{\mu}+(\boldsymbol{\Delta}, \boldsymbol{\Sigma} \mathbf{1}) \boldsymbol{\Omega}_{S}^{-1}\left(\mathcal{E}_{S}-\boldsymbol{\xi}_{S}\right)\right], \\
& =\mu_{i}+\mathcal{E}_{S 1}\left(\Delta_{i} \sigma_{S}^{2}+\sigma_{i S} \Delta_{S}\right)-\left(T C E_{S}\left(s_{\alpha}\right)-\mu_{S}\right)\left(\Delta_{i} \Delta_{S}+\sigma_{i S}\right),
\end{aligned}
$$

with $\mathcal{E}_{S 1}=\mathbb{E}\left[W_{1} \mid W_{1}>0, W_{2}>s_{\alpha}\right]$ and $\sigma_{i S}=\sum_{j=1}^{p} \sigma_{i j}$. Besides,

$$
\mathbb{E}\left[S \mid S>s_{\alpha}\right]=\mu_{S}+\mathcal{E}_{S 1} \sum_{i=1}^{p}\left\{\Delta_{i} \sigma_{S}^{2}+\sigma_{i S} \Delta_{S}\right\}-\left(T C E_{S}\left(s_{\alpha}\right)-\mu_{S}\right) \sum_{i=1}^{p}\left\{\Delta_{i} \Delta_{S}+\sigma_{i S}\right\} .
$$

\section{Conclusions}

In this paper, we proposed expressions to compute product moment of truncated multivariate distributions belonging to the selection elliptical family, showing in a clever way that their moments can be computed using an unique moment for their respective elliptical symmetric case. In contrast with other recent works, we avoid cumbersome expressions, having neat formulas for high-order truncated moments. To the best of our knowledge, this is the first proposal discussing the conditions of existence of the truncated moments for members of the selection elliptical family. Also, we propose optimized methods able to deal with extreme setting of the parameters, partitions with almost zero volume or no truncation.

We expect in the near future to use expressions in Section 6 to propose a robust likelihood-based censored regression model considering EST errors, able to fit multivariate censored responses with high skewness/kurtosis, presence of atypical observations and missing data. As more truncated moments for other symmetric elliptical distributions appear in the literature, we expect to implement the truncated moments for their respective asymmetric extended versions as well as censored models considering this last. Additionally, theoretical results can be extended to compute the moments of the class of extended generalized skew-elliptical distributions (see, [25]), where the jointly distributed condition in (44) is not longer considered.

Finally, theoretical and MC moments (among other functions of interest) for several multivariate asymmetric distributions are already available in our MomTrunc $\mathrm{R}$ package, which will be constantly updated when other treatable distributions are available.

\section{Acknowledgment}

Christian Galarza acknowledges support from FAPESP-Brazil (Grant 2015/17110-9 and Grant 2018/11580-1).

\section{References}

[1] James Tobin. Estimation of relationships for limited dependent variables. Econometrica: Journal of the Econometric Society, pages 24-36, 1958.

[2] G. M. Tallis. The moment generating function of the truncated multi-normal distribution. Journal of the Royal Statistical Society. Series B (Statistical Methodology), 23(1):223-229, 1961.

[3] B. G. Manjunath and Stefan Wilhelm. Moments calculation for the double truncated multivariate normal density. Available at SSRN 1472153, 2009.

[4] Saralees Nadarajah. A truncated bivariate t distribution. Economic Quality Control, 22(2):303-313, 2007.

[5] H. J. Ho, T. I. Lin, H. Y. Chen, and W. L. Wang. Some results on the truncated multivariate t distribution. Journal of Statistical Planning and Inference, 142:25-40, 2012.

[6] Juan C. Arismendi and Simon Broda. Multivariate elliptical truncated moments. Journal of Multivariate Analysis, 157:29-44, 2017.

[7] Raymond Kan and Cesare Robotti. On moments of folded and truncated multivariate normal distributions. Journal of Computational and Graphical Statistics, 25(1):930-934, 2017. 
[8] Roohollah Roozegar, Narayanaswamy Balakrishnan, and Ahad Jamalizadeh. On moments of doubly truncated multivariate normal mean-variance mixture distributions with application to multivariate tail conditional expectation. Journal of Multivariate Analysis, 177:104586, 2020.

[9] Ole Barndorff-Nielsen, John Kent, and Michael Sørensen. Normal variance-mean mixtures and z distributions. International Statistical Review/Revue Internationale de Statistique, pages 145-159, 1982.

[10] Wolfgang Breymann and David Lüthi. ghyp: A package on generalized hyperbolic distributions. Manual for $R$ Package ghyp, 2013.

[11] Reinaldo B Arellano-Valle and Marc G Genton. Multivariate extended skew-t distributions and related families. Metron, 68(3):201-234, 2010.

[12] A. Azzalini and A. Capitanio. Distributions generated and perturbation of symmetry with emphasis on the multivariate skew-t distribution. Journal of the Royal Statistical Society, Series B, 61:367-389, 2003.

[13] Reinaldo B Arellano-Valle, Máaarcia D Branco, and Marc G Genton. A unified view on skewed distributions arising from selections. Canadian Journal of Statistics, 34(4):581-601, 2006.

[14] Reinaldo B Arellano-Valle and Adelchi Azzalini. On the unification of families of skew-normal distributions. Scandinavian Journal of Statistics, 33(3):561-574, 2006.

[15] M. D. Branco and D. K. Dey. A general class of multivariate skew-elliptical distributions. Journal of Multivariate Analysis, 79:99-113, 2001.

[16] Raúl Alejandro Morán-Vásquez and Silvia L P Ferrari. New results on truncated elliptical distributions. Communications in Mathematics and Statistics, (53), 2019.

[17] L. A. Matos, M. O. Prates, M. H. Chen, and V. H. Lachos. Likelihood-based inference for mixed-effects models with censored response using the multivariate-t distribution. Statistica Sinica, 23:1323-1342, 2013.

[18] A. Dempster, N. Laird, and D. Rubin. Maximum likelihood from incomplete data via the EM algorithm. Journal of the Royal Statistical Society, Series B, 39:1-38, 1977.

[19] Michel Denuit, Jan Dhaene, Marc Goovaerts, and Rob Kaas. Actuarial theory for dependent risks: measures, orders and models. John Wiley \& Sons, 2006.

[20] Carlo Acerbi and Dirk Tasche. Expected shortfall: a natural coherent alternative to value at risk. Economic Notes, 31(2):379-388, 2002.

[21] Philippe Artzner, Freddy Delbaen, Jean-Marc Eber, and David Heath. Coherent measures of risk. Mathematical Finance, 9(3):203-228, 1999.

[22] Georg Ch Pflug. Some remarks on the value-at-risk and the conditional value-at-risk. In Probabilistic Constrained Optimization, pages 272-281. Springer, 2000.

[23] Ekaterina N Sereda, Efim M Bronshtein, Svetozar T Rachev, Frank J Fabozzi, Wei Sun, and Stoyan V Stoyanov. Distortion risk measures in portfolio optimization. In Handbook of portfolio construction, pages 649-673. Springer, 2010.

[24] Zinoviy Landsman and Emiliano A Valdez. Tail conditional expectations for elliptical distributions. North American Actuarial Journal, 7(4):55-71, 2003.

[25] Zinoviy Landsman, Udi Makov, and Tomer Shushi. Extended generalized skew-elliptical distributions and their moments. Sankhya A, 79(1):76-100, 2017. 
This figure "test.png" is available in "png" format from: http://arxiv.org/ps/2007.14980v1 